\title{
Critical Conditions of Dislocation Generation in Core-Shell Nanowires: A Review
}

\author{
A.M. Smirnov ${ }^{1}$, S.A. Krasnitckii ${ }^{1,2}$, S.S. $\operatorname{Rochas}^{1}$ and M.Yu. Gutkin $^{1,2,3}$ \\ ${ }^{1}$ School of Photonics, ITMO University, Kronverkskiy pr. 49, St. Petersburg, 197101, Russia \\ ${ }^{2}$ Institute for Problems in Mechanical Engineering, Russian Academy of Sciences, Bolshoj 61, Vasil. Ostrov, \\ St. Petersburg 199178, Russia \\ ${ }^{3}$ Higher School of Mechanics and Control Processes, Peter the Great St. Petersburg Polytechnic University, \\ Polytekhnicheskaya 29, St. Petersburg 195251, Russia
}

Received: August 03, 2020

\begin{abstract}
Dislocation-free core-shell nanowires are a promising material for quantum communication devices. The synthesis of such nanowires is associated with many expensive experiments. This review is devoted to analytical models aimed at predicting the critical conditions for the dislocation nucleation in core-shell nanowires and could be of interest to researchers dealing with elaboration and characterization of these nanowires.
\end{abstract}

\section{INTRODUCTION}

In recent years, much attention has been paid to nanowires (NWs) as a modern functional material for single photon devices - single photon detectors (SPDs), single photon emitters (SPEs), etc. - that are widely used in quantum communication technologies [1-3]. Most SPDs are based on avalanche detectors with Geiger mode. The avalanche breakdown process is due to the presence of a strong electric field required for electron and hole ionization. The carriers must travel a finite distance in the semiconductor layer under the influence of an electric field to gain a sufficient energy for ionization. This means that the ionization probability is negligible for a certain distance, the dead space [4,5]. For example, the ionization dead space in GaAs can be as long as 70 $\mathrm{nm}$ for an electric field of $5 \times 10^{7} \mathrm{~V} / \mathrm{m}$, that is more than twice the distance a carrier needs to achieve the ionization energy $[4,6]$. Reducing the size of the multiplication region to the thickness of the dead space in SPDs based on traditional planar materials leads to an improvement in the determination of the avalanche process and to a decrease the uncertainty during the avalanche multiplication [5]. From this prospect, semiconductor NWs pave the way for the further miniaturization of the multiplication region. In addition, the use of NWs in SPDs allows one to separate the absorption region and the multiplication region, increase the probability of photogeneration, and also reduces the afterpulse effect, i.e. reduces the erroneous repeated registration of a photon after its absorption [7-10].

It should be noted that the performance of SPDs depends not only on their design and physical properties of the device material, but also on their defect structure. For instance, in [11] it is shown that the presence of edge misfit dislocations (MDs) in $\mathrm{GaN}$ introduces multiple gap states that leads to a narrowing of the bandgap. Also, it is well known that electronic, magnetic, and optical properties of nanostructures greater depend on the presence of various defects in their structure than bulk materials. The defect structure of semiconductor NWs typically includes perfect and partial misfit dislocations [12], stacking faults [13], prismatic (PDLs) and glide (GDLs) dislocation loops and halfloops $[14,15]$. Modern experimental and theoretical methods have been extensively applied to the study of the dislocation-free NWs with high crystal structure syn-

Corresponding author:A.M. Smirnov, e-mail: smirnov.mech@gmail.com

(C) ITMO University, 2020 
thesis problem. Verified with experimental data theoretical models allow to define the critical parameters of the NWs that lead to defect generation. Thus, many researchers to date have focused on the development of theoretical models describing the defect structure of NWs.

Since the early 2000s, the first theoretical studies of misfit strains and mechanisms of their relaxation through generation of various defects in axially- [16-22] and radially-inhomogeneous (core-shell) [13,14,23-63] composite NWs have been published. A large part of these works was done within various continuum approaches $[21,23,24,26-$ $29,31,32,34,37,44-46,48-50,53,55,58,61-63]$ and aimed at the calculation of critical conditions of relaxation by defect generation. Referenced theoretical models can be divided into two groups. For both groups, the authors have used the energetic approach, considering the energy change due to the formation of the defects. To the first group we include the models, considering the nucleation of a final configuration of the defects, such as dislocations $[23,27,45,49]$, dislocation dipoles [32], dislocation loops [26,27,29,31,34,37,44,63]; to the second group we include the models, considering the energy barriers for nucleation and evolution of the defect configuration, such as dislocations and their dipoles [61], and dislocation loops [46,48,58,60-62].

\section{QUASI-EQUILIBRIUM ENERGY APPROACH FOR CALCULATING THE CRITICAL CONDITIONS OF MD GENERATION IN CORE-SHELL NWs}

Let us consider in more detail the quasi-equilibrium energy approach suggested for core-shell NWs in [23] and used in the models of the first group. In applying to MDs at interfaces in flat misfitting heterostructures like a thin epilayer on a semi-infinite substrate, this approach was invented by Frank and Van der Merwe [64] and adopted in the simplest macroscopic form by Matthews [65]. Since that time, many different modifications of this approach were suggested (see, for example, some papers [66-81] and books [82-84]). The authors of [23] used the approach variant suggested earlier by Gutkin and Romanov [67,73].

\subsection{Straight MDs parallel to the NW axis}

Within the model [23], the misfit accommodation process takes place in a composite core-shell NW by generation of a straight edge MD at the core-shell interface (Fig. 1). The energy approach consists in comparing the coherent strain energy of a MD-free NW $W_{1}$ being equal to the misfit strain energy $W^{f}$ relates to misfitting at the interphase boundary only, and the total energy of a NW with a MD generated at the interphase boundary $W_{2}$ that includes $W^{f}$, $W^{d}$ (the strain energy of the MD), $W^{c}$ (the energy of the MD core) and $W^{\text {int }}$ (the energy of elastic interaction of the MD and the misfit stress in the NW):

$$
\Delta W=W_{2}-W_{1}=W^{f}+W^{d}+W^{c}+W^{i n t}-W^{f}=W^{d}+W^{c}+W^{i n t}
$$

The expressions for $W^{d}, W^{c}$ and $W^{\text {int }}$ are given in [23] as follows:

$$
\begin{gathered}
W^{d}=\frac{D b^{2}}{2}\left(\frac{H(H-2)\left(H-r^{*}\right)\left(H-2-r^{*}\right)\left[2 H\left(H-2-r^{*}\right)-1+2 r^{*}\right]}{2\left[H^{2}-\left(2+r^{*}\right) H+r^{*}\right]^{2}}+\ln \frac{H\left(2-H+r^{*}\right)}{r^{*}}\right), \\
W^{c}=\frac{D b^{2}}{2}, \\
W^{\text {int }}=\sigma^{*} b r_{0} H(H-2),
\end{gathered}
$$

where $D=G /[2 \pi(1-v)], H=h / R, r^{*}=r_{c} / R$, and $\sigma^{*}=G f(1+v) /(1-v)$. Here $G$ is the shear modulus, $v$ is the Poisson ratio, $r_{c}$ is the dislocation core radius, $b$ is the magnitude of the MD Burgers vector, and $f$ is the misfit parameter. Note that, in considering straight MDs, the energy terms are given per unit length of the MD line.

The MD generation is energetically favorable, if it leads to a decrease in the total energy change: $\Delta W<0$. In order to define the critical conditions of a relaxation process, one have to solve the equation $\Delta W=0$ for the NW parameter, for example, for the shell thickness $h$ or for the misfit parameter $f$. Furthermore, misfit parameter $f$ can be defined as follows: $f=2\left(a_{c}-a_{s}\right) /\left(a_{c}+a_{s}\right)$, where $a_{c}$ and $a_{s}$ are the lattice parameters of the core and shell materials, correspondingly. 
In the case of a NW with thin shell $\left(h<<r_{0}\right)$, the equation $\Delta W=0$ takes the form:

$$
1-\frac{2 h\left(h-r_{c}\right)}{\left(2 h-r_{c}\right)^{2}}+\ln \frac{2 h-b}{r_{c}}=8 \pi(1+v) f \frac{h}{b}
$$

which coincides with that found earlier in the case of a thin flat epilayer on a semi-infinite substrate [66].

Fig. 2 illustrates the dependence of the critical shell thickness $h_{c}$ on the misfit parameter $f$ for $v=0.3$ and $r_{c}=b=$ $0.4 \mathrm{~nm}$ at $h \ll<r_{0}$. Herein the equation $\Delta W=0$ (see Eq. (5)) was solved with respect to $h$, as a result $h_{c}$ was found. The generation of MD is energetically favorable in $\beta$-area of the Fig. 2, when the total energy change $\Delta W$ is less than zero. Therefore, the critical thickness $h_{c}$ is the shell thickness above which the nucleation of MD is energetically favorable. The MD generation in the $\alpha$-area is unfavorable, since the total energy change $\Delta W$ is positive there. An increase in the misfit parameter $f$ leads to an increase in the level of misfit stresses in the NW and to a decrease in the critical thickness of the shell $h_{c}$. Thus, using Fig. 2, it is possible to select the parameters of the NWs (materials and shell thickness) for synthesis of MD-free NWs with coherent boundary between the core and the shell.

In the opposite limiting case of a thin core surrounded by a thick shell, $h \gg>r_{0}$, the generation of a MD becomes energetically favorable if $h<b \exp \left[4 \pi(1+v) f r_{0} / b+1 / 2\right]$, see Ref. [23].

Thus, the authors [23] showed for the first time, that, in the general case of comparable core radius $r_{0}$ and shell thickness $h$, the MD generation should be expected when the shell thickness is not either extremely small nor extremely large: $h_{c 1}<h<h_{c 2}$. This result is clearly issued from the analyses of another critical parameter of the system, namely, the critical misfit value $f_{c}$ which is easily extracted from the inequation $\Delta W<0$ as follows [23]: $f>f_{c}$, where

$$
f_{c}=\frac{b}{4 \pi(1+v) r_{0} H(2-H)}\left(1+\frac{H(H-2)\left(H-r^{*}\right)\left(H-2-r^{*}\right)\left[2 H\left(H-2-r^{*}\right)-1+2 r^{*}\right]}{2\left[H^{2}-\left(2+r^{*}\right) H+r^{*}\right]^{2}}+\ln \frac{H\left(2-H+r^{*}\right)}{r^{*}}\right)
$$

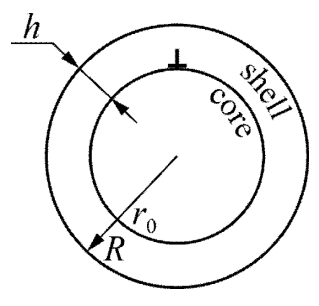

Fig. 1. Schematic representation of a core-shell NW cross section with a straight $\mathrm{MD} ; r_{0}$ is the core radius, $R$ is the NW outer radius, $h$ is the shell thickness.

(a)

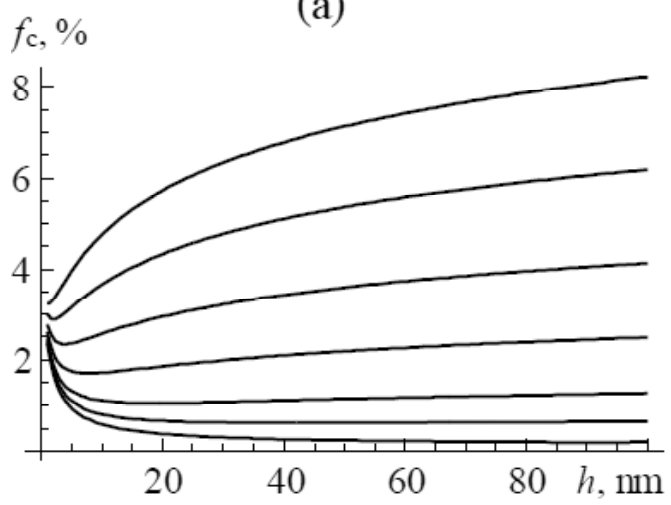

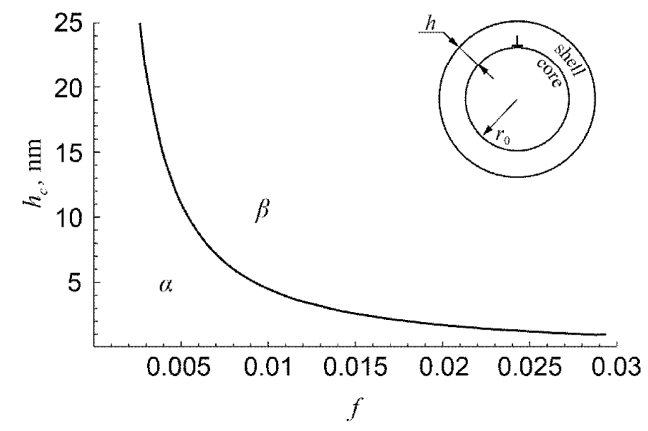

Fig. 2. The dependence of the critical shell thickness $h_{c}$ on the misfit parameter $f$ in the thin-shell approximation $h \ll r_{0}$. The Figure is a sketch of Fig. 4 from the work [23], all major designations are saved.

(b)

$f_{\mathrm{c}}, \%$

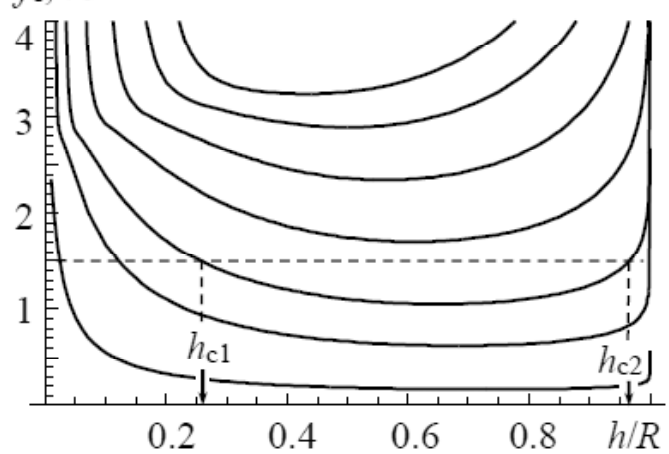

Fig. 3. Dependence of the critical misfit $f_{\mathrm{c}}$ on (a) the shell thickness $h$ and (b) the ratio $h / R$ for the following values of the core radius $r_{0}$ (from top to bottom): $r_{0}=1.5,2,3,5,10,20$, and $100 \mathrm{~nm}$. Adapted from [85]. 
The dependence of the critical misfit $f_{c}$ on the characteristic dimensions of the NW at $v=0.3$ and $b=0.4$ $\mathrm{nm}$ is shown in Fig. 3 [85]. As is seen, at a very small core radius $r_{0}=1.5 \mathrm{~nm}, f_{c}$ is rather large, $>3 \%$, and monotonically increases with increasing the shell thickness $h$. At a slightly larger core radius, $r_{0}=2 \mathrm{~nm}$, a minimum appears on the $f_{c}(h)$ curve at $h \approx 2 \mathrm{~nm}$, and the curve itself goes much lower. As $r_{0}$ further increases, this minimum deepens, smoothes and shifts to the re-

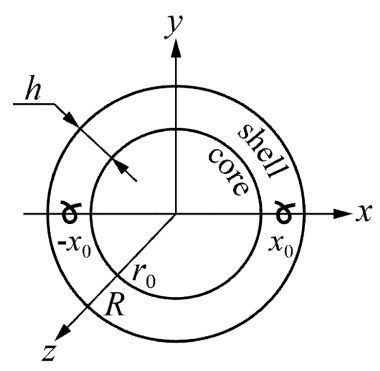

Fig. 4. Schematic representation of a core-shell NW cross section with a dipole of screw MDs placed at the $\pm x_{0}$ positions in the shell. The Burgers vectors and lines of the MDs are parallel to the $z$-axis of the cylindrical NW.

(a)

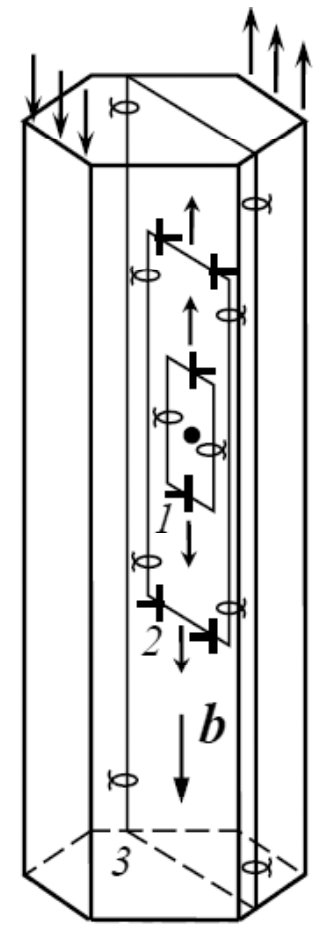

(b)

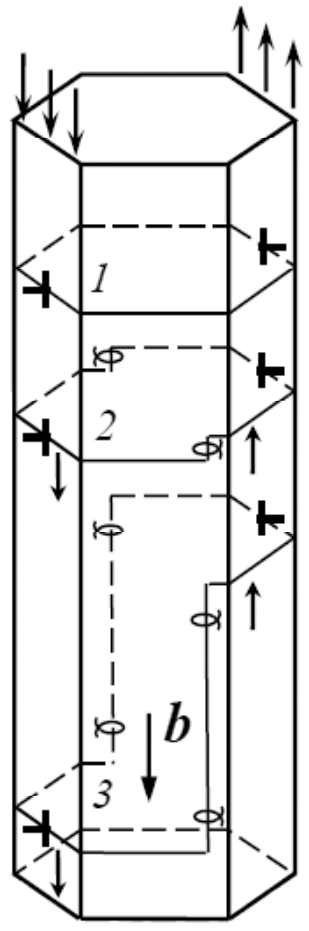

(c)

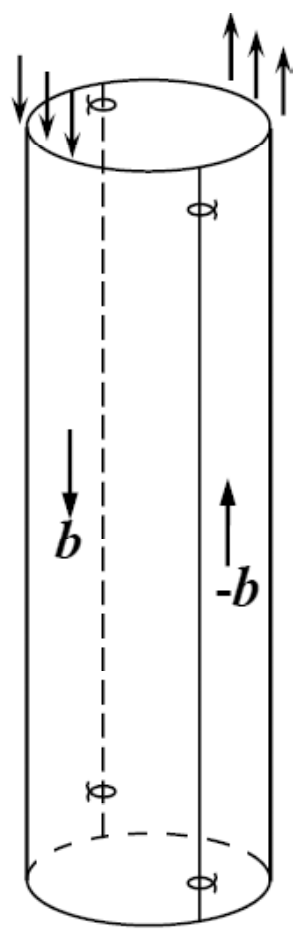

Fig. 5. Sketch of possible mechanisms of screw misfit dislocation dipole formation at the NW/matrix interface when the NW is under antiplane eigenstrain. (a) Generation of a rectangular glide dislocation loop with Burgers vector $\boldsymbol{b}$, which expends in a longitudinal section of the NW from a stress concentrator (black point) in such a way that the two screw segments gradually transform into the two straight screw dislocations while the edge segments glide to the ends of the NW. (b) Extension of a pre-existing prismatic dislocation loop with Burgers vector $\boldsymbol{b}$, which also produces a dipole of elongated screw dislocation segments, while the prismatic semi-loops glide to the ends of the NW. (c) The calculation model of a cylindrical nanowire with a dipole of screw misfit dislocations with Burgers vectors $\pm \boldsymbol{b}$, placed at the NW/matrix interface. In (a) and (b), the numbers 1, 2, and 3 indicate the subsequent stages of the dipole formation. Adapted from [86]. 
$h_{c 1}<h<h_{c 2}$. For example, for $f=1.5 \%$ and $r_{0}=10 \mathrm{~nm}$, the plot in Fig $3 \mathrm{~b}$ gives $h_{c 1} \approx 0.26 R \approx 3.5 \mathrm{~nm}$ and $h_{c 2} \approx 0.97 R \approx 323$ $\mathrm{nm}$, i.e., the coherent state turns out to be energetically favorable at either a very thin or very thick shell. In the second case, for example, for the same $f$ and $r_{0}=5 \mathrm{~nm}$, there is no intersection with the $f_{c}(h / R)$ curve, which means the energetic preference of the coherent state at any shell thickness.

It is worth noting that similar approaches were used to investigate the critical conditions for the onset of misfit stress relaxation in core-shell NWs through generation of straight wedge disclinations, wedge disclination dipoles and walls of equidistant edge dislocations [24].

Besides edge MDs and wedge disclinations, screw MDs were also analyzed in detail. For example, Wang et al. [32] considered the misfit-stress relaxation process in a core-shell NW through the generation of a dipole of screw MDs (see Fig. 4). In this model, the core and the shell were treated as elastically different materials, and $\Gamma$ was chosen as a convenient parameter that determines the difference in their elastic properties: $\Gamma=2 G_{s} /\left(G_{c}+G_{s}\right)$, where $G_{c}$ and $G_{s}$ are the shear moduli of the core and the shell materials, correspondingly.

Shodja et al. [86] suggested two possible mechanisms for the formation of such a dipole of screw MDs at the $\mathrm{NW} /$ matrix interface in the case when the NW is embedded to an infinite matrix and subjected to anti-plane eigenstrain (Fig. 5). Similar mechanisms could be responsible for the generation of the screw MD dipole in core-shell NWs as well.

The authors of the work [32] define an equilibrium position of the MDs dipole from the condition $\boldsymbol{F}=\mathbf{0}$, where $\boldsymbol{F}$ is the Peach-Koehler force acting on the positive MD (at $x=x_{0}$ ):

$$
\begin{gathered}
F_{x}=\frac{\pi r_{0} \sigma_{z y}}{G_{s} b}\left(\frac{1-\Gamma}{\tilde{x}^{4}-1} \tilde{x}-\frac{1}{4 \tilde{x}}+\sum_{n=1}^{\infty} \frac{\tilde{x}^{2(2 n-1)}-(1-\Gamma)^{2} \tilde{x}^{-2(2 n-1)}}{\tilde{x}\left(1-\Gamma+\tilde{R}^{2(2 n-1)}\right)}\right), \\
F_{y}=F_{z}=0, \\
\sigma_{z y}+i \sigma_{z x}=G_{s}\left(\frac{\sigma_{z y}+i \sigma_{z x}=G_{c} \Gamma\left(\frac{b x_{0}}{\pi\left(z^{2}-x_{0}^{2}\right)}+\sum_{n=1}^{\infty}(2 n-1) A_{n} z^{2(n-1)}\right) \text { at }|z|<r_{0},}{\pi\left(z^{2}-x_{0}^{2}\right)}+\frac{(1-\Gamma) b r_{0}^{2} x_{0}}{\pi\left(x_{0}^{2} z^{2}-r_{0}^{4}\right)}+\sum_{n=1}^{\infty}(2 n-1) A_{n}\left[z^{2(n-1)}-(1-\Gamma) r_{0}^{2(2 n-1)} z^{-2 n}\right]\right) \text { at } r_{0}<|z|<R, \\
A_{n}=\frac{b}{\pi} \frac{x_{0}^{2 n-1}+(1-\Gamma) r_{0}^{2(2 n-1)} x_{0}^{-(2 n-1)}}{(2 n-1)\left[R^{2(2 n-1)}+(1-\Gamma) r_{0}^{2(2 n-1)}\right]},
\end{gathered}
$$

where $\tilde{x}=x_{0} / r_{0}, \tilde{R}=R / r_{0}$. In numerical calculations, the series were truncated at $n=30$ for a relative error below $1 \%$.

Based on the dependences of the Peach-Koehler force on the position of MDs, the authors showed that, when the shell thickness $h$ is above the critical value $h_{c}$ there exists equilibrium positions for the MDs dipole $\left(x_{0}=1.3 r_{0}\right.$ and $\left.2.25 r_{0}, h=4 r_{0}\right)$, but the stable position is when the MDs are close to the core-shell interface $\left(x_{0}=1.3 r_{0}\right)$. When the shell thickness $h$ is equal to its critical value $h_{c}$, the two equilibrium positions of the MDs dipole converge to a single equilibrium position $\left(x_{0}=1.5 r_{0}, h=h_{c}=3 r_{0}\right)$. There is no equilibrium position for the MDs dipole when the shell thickness $h$ is less than the critical value $h_{c}$.

Fig. 6 shows the dependence of the critical shell thickness $h_{c}$ on the parameter $\Gamma$. As is seen, $h_{c}$ monotonically decreases with $\Gamma$. In the $\alpha$-area, there is always no equilibrium position for the MDs dipole, but in the $\beta$-area, there are two equilibrium positions for the MDs.

In the aforementioned theoretical models of MDs in core-shell NWs, the authors considered the misfit and MD stresses within the classical theory of elasticity. However, in core-shell NWs with thinnest cores and/or shells, the effects of the surface/interface energies, strains and stresses may occur rather strong. One of the continuum approaches, which address the surface/interface effects on elastic behavior of solids, is the so-called surface/interface elasticity which is based on the concepts of surface free energy and surface stress in solids, first introduced by Gibbs [87] and developed gradually over the time [88-95] (see also Ref. [95] for more references). In particular, Gurtin and Murdoch $[89,90]$ formulated the framework for solving problems in the surface/interface elasticity. In the framework of this approach, the surface/interface is considered as an atomically thin layer with elastic characteristics differing from those of the bulk material [96-98]. 


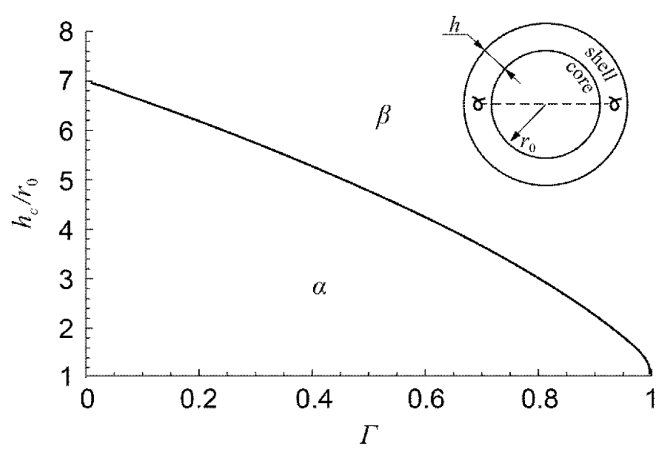

Fig. 6. The dependence of the critical shell thickness $h_{c}$ on the parameter $\Gamma$. The Figure is a sketch of Fig. 5 from the work [32], all major designations are saved.

Zhao et al. [45] and Enzevaee et al. [49] analyzed the critical conditions for the onset of either an individual edge MD (model 1) [45,49] or a dipole of such MDs (model 2) [49] at the interface of an elastically isotropic and inhomogeneous core-shell NW within both the classical and surface/interface elasticity approaches with special attention to the non-classical surface/interface effect. In particular, Enzevaee et al. [49] showed that this effect can be significant for very fine cores of radius smaller than roughly 20 interatomic distances $(\sim 5-7 \mathrm{~nm})$. The positive (negative) surface/interface Lamé constants mostly make the generation of MDs easier (harder). On the contrary, the positive (negative) residual surface/interface tensions mostly make their generation harder (easier). It was also showed that the formation of an individual MD is energetically more preferential in finer two-phase NWs, while the formation of a MD dipole is more expectable in the coarser ones. Fig. 7 illustrates the non-classical surface/interface effect on the critical misfit value $f_{c}$ within models 1 and 2 .

Shodja et al. [86] investigated similar effect for the generation of a dipole of screw MDs at the interface between a NW with uniform shear misfit strain (Fig. 5) and its surrounding unbounded matrix. The analysis was carried out within the surface/interface elasticity theory with using the complex potential variable method. It was shown that the critical radius of the NW corresponding to the onset of the MD dipole generation decreases with the increase in the uniform shear eigenstrain inside the NW as well as when the stiffness of the NW increases with respect to the matrix. Moreover, the critical radius strongly depends on the non-classical interface parameter $\gamma=\left(\mu_{s}-\tau_{s}\right) / G_{m}$, where $\mu_{s}$ is the shear modulus of the $\mathrm{NW} /$ matrix interface, $\tau_{s}$ is the residual interface tension, and $G_{m}$ is the shear modulus of the matrix. The non-classical interface effect is that positive (negative) values of the interface parameter $\gamma$ significantly increase (decrease) the NW critical radius with respect to the classical result. This effect becomes stronger with increase of matrix stiffness.

\subsection{Circular prismatic loops of MDs in core-shell NWs}

The critical conditions for the formation of circular prismatic dislocation loops (PDLs) in core-shell NWs were analyzed in a number of works [26,27,29,31,34,37,44, $50,63]$. Some authors operated with their own original [26,28,29,31,63] or already known [34,50] strict solutions for circular PDLs in elastic cylinders, while the others dealt with approximate formulas for strain energies of PDLs $[27,37,44]$.

The first strict solution was done by Ovid'ko and Sheinerman [26] with using the classical methods of solving the boundary-value problems in the theory of elasticity [99]. It was later reproduced in review [28] and some books $[84,85,100]$, so we do not consider it here in detail. The authors calculated the energy difference $\Delta W$ (given by Eq. 1) caused by the generation of a closed circular PDL in an elastically homogeneous core-shell NW (Fig. 8) and extracted a solution for the critical misfit value $f_{c}$ from the equation $\Delta W=0$.

Fig. 9 illustrates the dependence of $f_{c}$ on the shell thickness $h$ and core radius $r_{0}$. As is seen, $f_{c}$ decreases monotonically with increasing $h$ and $r_{0}$. This means that, for a given misfit $f$, the nucleation of a PDL becomes energetically favorable if $h$ and $r_{0}$ reach some critical values. For comparison, in Fig. 9a, the $f_{c}(h / b)$ dashed curves for straight MDs considered in [23] (see the previous section), are shown. In the case of small $h$, these curves pass below the solid curves plotted for PDLs, which means the energetic preference of straight MDs at the initial stages of shell growth. The intersections of the dashed curves with the solid ones correspond to an equal gain in energy from the formation of a straight MD or a PDL. For example, for a core radius $r_{0}=10 b \approx 4$ $\mathrm{nm}$, this balance is achieved at a shell thickness $h \approx 30 b \approx 12 \mathrm{~nm}$. For larger shell thicknesses, the formation of PDLs becomes more profitable. It is important to note that, if the shell thickness is more than 3-4 times greater than the core radius, then the appearance of PDLs can be favorable even for those values of $f$ and $r_{0}$, at which the formation of straight MDs is not [26].

Aifantis et al. [29] proposed a similar model (see Fig. 8), however, by using their own solutions for the misfit stresses and the stress fields of the circular PDL in an elastically inhomogeneous core-shell NW. The latters were found by the method of virtual surface defects (see, for example, reviews [101,102] for more references and details). In the limit of elastically homogeneous coreshell NW, the authors [29] obtained the following energy terms figuring in Eq. (1): 
Model 1
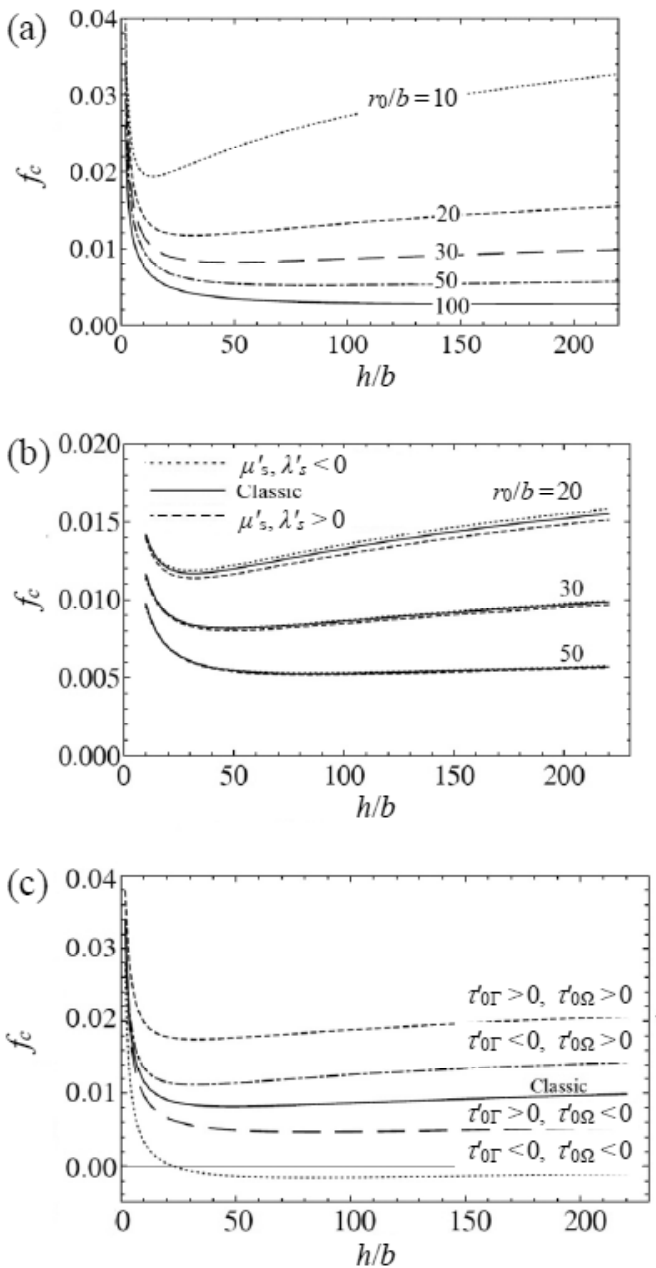

Model 2
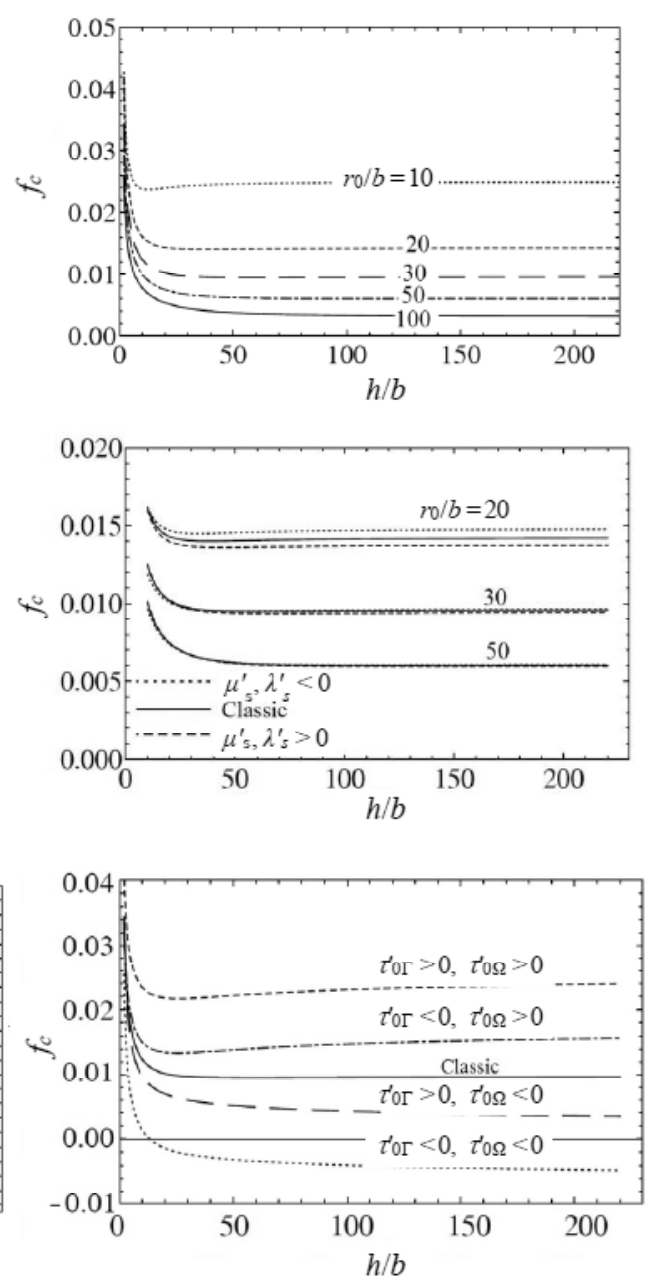

Fig. 7. Dependence of the critical misfit strain $f_{c}$ on the normalized shell thickness $h / b$ at different values of the normalized core radius $r_{0} / b$ and shear moduli ratio $G_{s} / G_{c}=1.5$ for different normalized surface/interface Lamé constants $\mu_{s}^{\prime}=\lambda_{s}^{\prime}= \pm 0.1 \mathrm{~nm}$ and zero normalized residual surface/interface tensions $\tau_{0 i}^{\prime}$ within (a) the classical and (b) the classical and non-classical solutions. (c) Comparison of the classical and non-classical solutions for different signs of the surface/interface residual tensions at $r_{0}=30 b, G_{s} / G_{c}=1.5$, and $\mu_{s}^{\prime}=\lambda_{s}^{\prime}=0$. Here $G_{s}$ and $G_{c}$ are the shell and core shear moduli; $\mu_{s i}^{\prime}=\mu_{s i} / G_{c}, \lambda_{s i}^{\prime}=\lambda_{s i} / G_{c}, \tau_{0 i}^{\prime}=\tau_{0 l} / G_{c}$, where $i=\Gamma, \Omega ; \mu_{s i}$ and $\lambda_{s i}$ are the surface/interface Lamé constants, $\tau_{0 i}$ is the residual surface/interface tension, $\Gamma$ and $\Omega$ designate the core/shell interface and the shell surface, respectively. Adapted from [49].

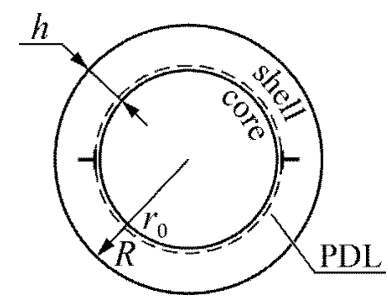

Fig. 8. Schematic representation of a core-shell NW cross section with a circular PDL.

$$
\begin{gathered}
W^{d}+W^{c}=\frac{G b^{2} r_{0}}{2(1-v)} \ln \frac{1.08 \alpha r_{0}}{b}-\frac{G b^{2} r_{0} t}{1-v} \int_{0}^{\infty} Q(p) \mathrm{d} p, \\
W^{\text {int }}=-\frac{2 \pi G b f(1+v)\left(1-t^{2}\right)}{1-v} r_{0}^{2},
\end{gathered}
$$

where the parameter $\alpha$ accounts for the dislocation core energy (for metals $\alpha=1$ ), $t=r_{0} / R$, and the integrand $Q(p)$ is 
(a)

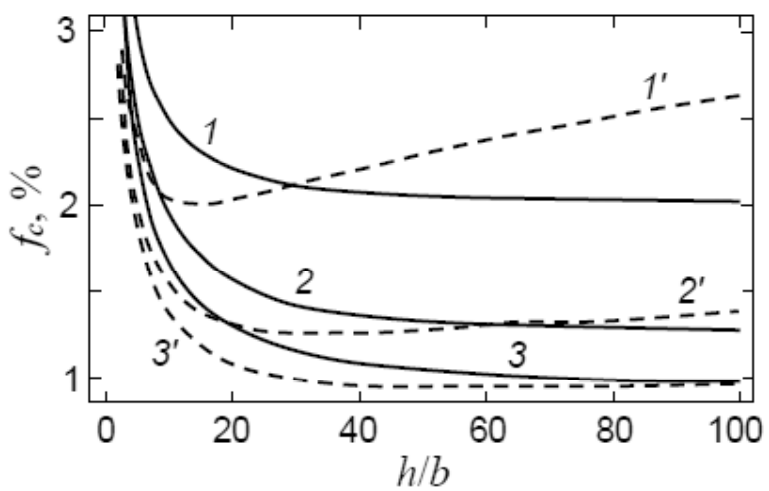

(b)

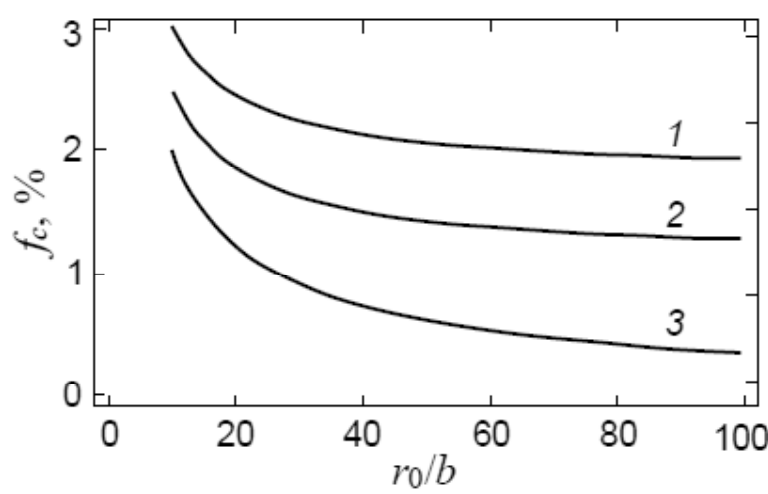

Fig. 9. Dependence of the critical misfit $f_{c}$ on the shell thickness $h$ and core radius $r_{0}$ for the Poisson ratio $v=0.3$ and the dislocation core radius $r_{c}=b$. (a) Dependence of $f_{c}$ on $h$ at $r_{0} / b=10$ (curves $\left.1,1^{\prime}\right), 20\left(2,2^{\prime}\right)$ and $30\left(3,3^{\prime}\right)$. (b) Dependence of $f_{c}$ on $r_{0}$ at $h / b=5$ (curve 1), 10 (2) and $\infty$ (3). The solid curves correspond to the generation of a PDL, the dashed curves to that of a straight MD. Adapted from [26].

$Q(p)=\frac{\tilde{t}\left[\left(p I_{0} A_{1}+I_{1} A_{2}\right) B_{1}+p^{3}\left(t I_{0}^{*} A_{3}+I_{1}^{*} A_{4}\right) B_{2}\right]}{T}$

with

$T=t\left[p^{2} I_{0}^{2}-\left(p^{2}-2 v+2\right) I_{1}^{2}\right] \cdot\left[\left(p^{2}+4\right) K_{1}^{2}-p^{2} K_{0}^{2}\right]$

$A_{1}=p t I_{0}^{*}\left[\left(p^{2}+4\right) K_{1}^{2}-p^{2} K_{0}^{2}\right]+I_{1}^{*}\left[-2 p^{2} K_{0}^{2}\right.$

$\left.+p\left(p^{2}(t-1)-2(1+v)\right) K_{1} K_{0}+2\left(t p^{2}-2 v+2\right) K_{1}^{2}\right]$,

$A_{2}=2 p t I_{0}^{*}\left[p^{2} K_{0}^{2}+p(1+v) K_{1} K_{0}\right.$

$\left.-\left(p^{2}-2 v+2\right) K_{1}^{2}\right]+I_{1}^{*}\left[-2(t-1) p^{3} K_{0} K_{1}\right.$

$\left.+\left(p^{2}-2 v+2\right) p^{2} K_{0}^{2}-\left(p^{2}+4\right)\left(t p^{2}-2 v+2\right) K_{1}^{2}\right]$,

$A_{3}=I_{1}\left[p^{2} K_{0}^{2}-\left(p^{2}-2 v+2\right) K_{1}^{2}\right]$,

$A_{4}=I_{0}\left[-p^{2} K_{0}^{2}+\left(t p^{2}-2 v+2\right) K_{1}^{2}\right]-p^{2}(t-1) I_{1} K_{0} K_{1}$,

$B_{1}=-p I_{1}^{* *} K_{0}+p \tilde{t} I_{0}^{* *} K_{1}+\tilde{t}$

$B_{2}=I_{1}^{* *} K_{1}-\tilde{t}_{2}^{* * *} K_{2}$.

Here $I_{0,1}$ and $K_{0,1,2}$ are the modified Bessel functions of argument $p, I_{0,1}^{*}$ are the modified Bessel functions of argument $t p$, and $I_{0,1,2}^{* *}$ are the modified Bessel functions of argument $\tilde{t} p ; \tilde{t}=\left(r_{0}-r_{c}\right) / R$, and $r_{c}=b / \alpha$.

Finally, with taking into account Eqs. (1) and (12)(14), the solution of the equation $\Delta W=0$ was given as follows [29]:

$$
h_{c}=r_{c}\left(\frac{1}{\sqrt{1-U}}-1\right), U=\frac{b \ln \left(1.08 \alpha r_{0} / b\right)}{4 \pi(1+v) f r_{0}}
$$

Fig. 10 illustrates the dependence of the critical shell thickness $h_{c}$ on the misfit strain $f$. In these calculations, the radius of the PDL was equal to the core radius $r_{0}$, $r_{0}=40 \mathrm{~nm}$. The asymptote shows the critical misfit strain for the mismatched cylindrical inclusion in an infinite medium $(R \rightarrow \infty)$. PDL generation is energetically favorable in the $\beta$-area and unfavorable in the $\alpha$-area.

Colin [31] extended the theoretical consideration of the circular PDL formation in a core-shell NW to the case when the elastically isotropic core and shell have different elastic moduli. Although this work contains a description of some principal stages of the solution procedure, but not its final result in any transparent form suitable for checking, one can understand that the author used a classical way similar to that utilized in Ref. 26. In the framework of a thermodynamic equilibrium approach, he calculated the energy variation from the dislocation-free NW and determined the equilibrium

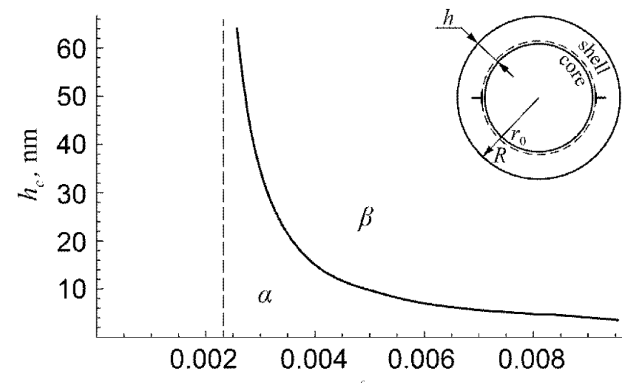

Fig. 10. The dependence of the critical shell thickness $h_{c}$ on the misfit strain $f$. The core radius is $r_{0}=40 \mathrm{~nm}$, the radius of the PDL equals to the core radius $r_{0}$. The asymptote shows the critical misfit strains for the mismatched cylindrical inclusions in an infinite medium $(R \rightarrow \infty)$. The Figure is a sketch of Fig. 4 from the work [29]. 
(a)

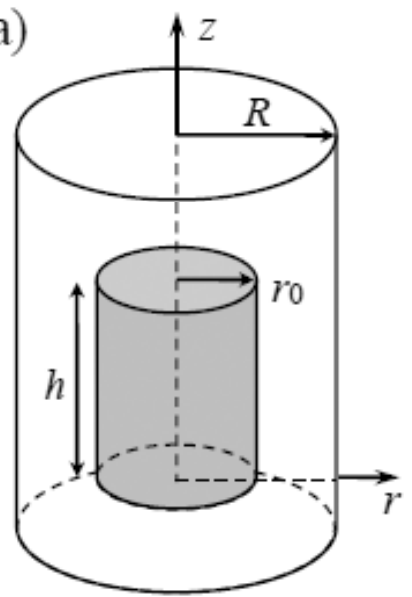

(b)

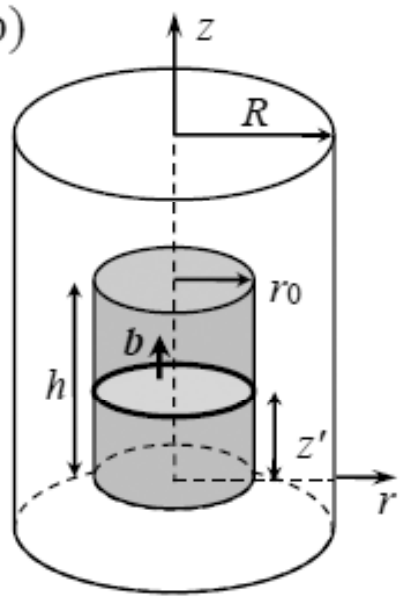

(c)

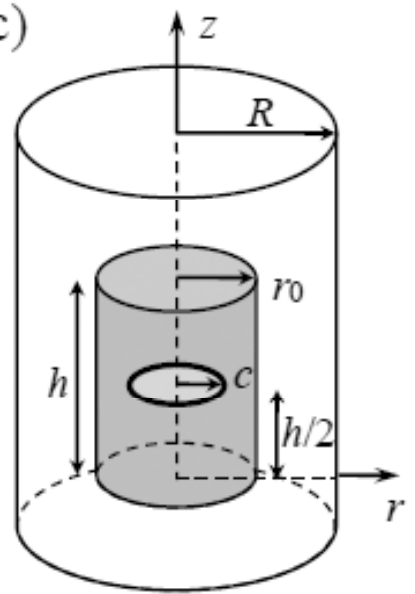

Fig. 11. Mechanisms of misfit stress relaxation in a NW of radius $R$ containing a coaxial finite cylindrical core of radius $r_{0}$ and length $h$. (a) Defect-free NW. (b) A PDL with Burgers vector $\boldsymbol{b}$ forms around the core in the NW. (c) Penny-shape mode I crack of radius $c$ nucleates in the middle cross section of the core. Adapted from [34].

(a)

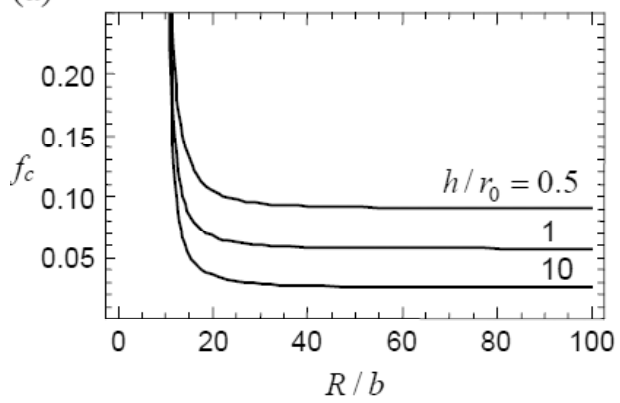

(b)

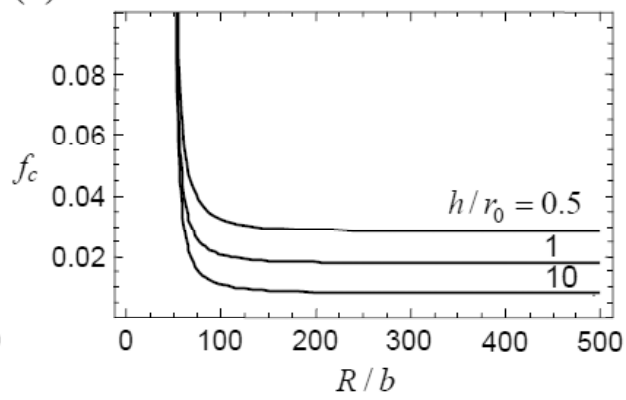

Fig. 12. Dependence of the critical misfit $f_{c}$ on the normalized NW radius $R / b$ for various values of $h / r_{0}$ and $r_{0} / b=10$ (a) and 50 (b). Adapted from [34].

position of the PDL as a function of the misfit stress, the nanowire radii, and the ratio of the shear moduli between the two phases. The main conclusion was that, depending on misfit strain and radii, for a sufficiently soft core with respect to the shell, PDLs may form within the core with equilibrium positions located at a few interatomic distances from the core/shell interface. Earlier, this effect of the so-called 'stand-off' positions of MDs was extensively discussed for planar interfaces (see some original papers [103-105] and book [84]).

It is worth noting that the solutions [26] for the stress fields and strain energy of a PDL in an elastic cylinder was later used by Gutkin et al. [34] and Colin [50] in their further extensions to the cases of PDL generation in NWs with cores of finite length [34] and in three-layer NWs [50]. In both the cases, the composite NWs were supposed elastically homogeneous.

Let us briefly consider the results reported by Gutkin et al. [34]. In this case, the stress fields [26] of a PDL were utilized to find the misfit stress fields of the finitelength core which was modeled as an inclusion with one-dimensional (1D) dilatation eigenstrain. The stress fields of the inclusion was calculated by integration of the stress fields of axial virtual PDLs with infinitesimal Burgers vectors continuously distributed over the side surface of the core. Two different mechanisms of misfit stress relaxation in such a NW with a cylindrical inclusion were proposed and analyzed in [34]. The first mechanism involved the formation of a PDL around the inclusion while the second mechanism suggested the formation of a penny-shaped crack in the inclusion cross section (Fig. 11). It was shown that both the mechanisms can be realized in wide ranges of the structural and geometric parameters of the NW and inclusion.

For example, Fig. 12 shows the dependence of the critical (1D) misfit $f_{c}$ on the NW radius $R$ for $r_{\mathrm{c}}=b, v=0.3$, and various values of the core sizes $h$ and $r_{0}$. The plots are shown for the case $z^{\prime}=h / 2$, where the PDL is located in the middle cross section of the core. In this cross section, the misfit stress $\sigma_{z z}^{f}$ is commonly maximum, and so the PDL provides the strongest relaxation of misfit stresses. As is seen in Fig. 12, for given inclusion sizes $h$ and $r_{0}$, the critical misfit $f_{c}$ first rapidly decreases with an increase in the NW radius $R$ and then (when $R$ becomes several times as large as the core radius $r_{0}$ ) approaches a constant value. This constant value of $f$ 


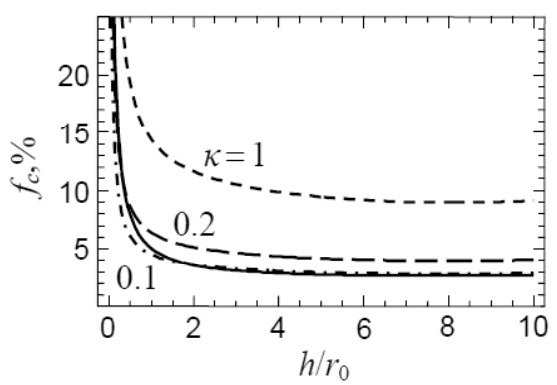

Fig. 13. Dependence of the critical misfit $f_{c}$ for the formation of PDL (solid curve) and crack (dashed curves) on the normalized core length $h / r_{0}$, for $r_{0}=15 b, R=30 b$ and different values of $\kappa$. Adapted from [34].

corresponds to the limiting case of a finite-length cylindrical inclusion in an infinite matrix. Similarly, for specified NW and core radii $R$ and $r_{0}, f_{c}$ decreases with an increase in the inclusion length $h$. At large values of the core aspect ratio $h / r_{0}\left(h / r_{0}>10\right)$, the dependence of $f_{c}$ on $h$ approaches a constant level, which depends on the values of $R$ and $r_{0}$. This level corresponds to the limiting case of a PDL in a core-shell NW [26,29].

The critical conditions of crack initiation inside the core (see Fig. 11c) were investigated using the force criterion $K_{1}>K_{1 c}$, where $K_{1}$ is the crack stress intensity factor and $K_{1 c}$ is the critical value of this coefficient for a brittle material. The authors [34] showed that at a sufficiently large misfit strain $f$, this mechanism becomes effective if the radius of the opened crack $c$ falls into the interval $c_{1}<c<c_{2}$, the width of which depends on the geometric dimensions of the core and on their relation to the radius of the NW. At the same time, in most cases, the initiation of such a crack turns out to be less preferable than the formation of a PDL, since it requires a higher critical mismatch $f_{c}$ (Fig. 13). An exception is the situation when the length of the core is small (less than its diameter), and the specific energy of the free surface of the core material $\gamma \approx \kappa G b / 8$ is an order of magnitude lower than the usual values of the surface energy $(\kappa \approx 1)$. Under these conditions, the crack becomes more preferable than the PDL.

Recently, Chernakov et al. [63] revisited the problem which was earlier considered in [26,29]. First, they solved the boundary-value problem in the classical theory of elasticity for a circular PDL in an elastically isotropic cylinder by the Lurie method [106] different from those [26,29] used before for solving this problem. Based on this solution, both the self energy $\left(W^{d}+W^{c}\right)$ of the PDL and the interaction energy $\left(W_{i n t}^{d d}\right)$ for a pair of such PDLs were found as follows [63]:

$$
\begin{gathered}
W^{d}+W^{c}=\frac{G b^{2} r_{0}}{2(1-v)}\left(\ln \frac{1.08 \alpha r_{0}}{b}-2 t \int_{0}^{\infty} \Theta(\beta, t) \mathrm{d} \beta\right), \\
W_{i n t}^{d d}=\frac{G b^{2} r_{0}}{1-v}\left(\pi \int_{0}^{\infty} J_{1}^{2}(\kappa)\left(1+\frac{h}{r_{0}} \kappa\right) e^{-\frac{h}{r_{0}}} \mathrm{~d} \kappa-2 t \int_{0}^{\infty} \Theta(\beta, t) \cos (\beta \tilde{h}) \mathrm{d} \beta\right),
\end{gathered}
$$

where

$$
\Theta(\beta, t)=\frac{\beta^{2} t^{2} I_{0}^{*_{2}}+w I_{1}^{*_{2}}-2 \beta t I_{1}^{*} I_{0}^{*}\left(w I_{1} K_{1}+\beta^{2} I_{0} K_{0}\right)}{\beta^{2} I_{0}^{2}-w I_{1}^{2}},
$$

$I_{0,1}^{*}=I_{0,1}(t \beta), I_{0,1}=I_{0,1}(\beta), K_{0,1}=K_{0,1}(\beta)$ and $I_{0,1}(\beta)$ and $K_{0,1}(\beta)$ are the modified Bessel functions of the first kind and the Macdonald functions, correspondingly; $J_{1}(\kappa)$ is the Bessel function of the first kind, $w=\beta^{2}-2 v+2, t=r_{0} / R$, $\tilde{h}=h / R$, and $h$ is the distance between the PDLs. It is seen that Eq. (16) with the integrand $\Theta(\beta, t)$ is much simpler than earlier formulas for the PDL energy in [26,29] (see, for example, Eqs. (12) and (14) with corresponding designations from [29]).

With these results on hand, Chernakov et al. [63] calculated the critical conditions for nucleation of a circular PDL in a core-shell NW and found results similar to those in [26,29]. Moreover, they also calculated the change in the total energy of the core-shell NW being in a partly relaxed state, with an infinite row of PDLs periodically distributed at the core-shell interface along the NW axis, per unit length of the NW. Minimizing this energy change by the row period, they found the equilibrium distance between the PDLs, compared it with results of direct experimental observations of MDs in InAs-GaAs core-shell NWs [36], and showed that their theoretical results (8.35-9.05 nm) well corresponded to the experimental data $(7.0-8.5 \mathrm{~nm})$.

Thus, the models of the first group considered in this section allowed to calculate the critical conditions for the formation of either straight MDs or closed circular misfit PDLs in core-shell NWs. The comparison of these condi- 


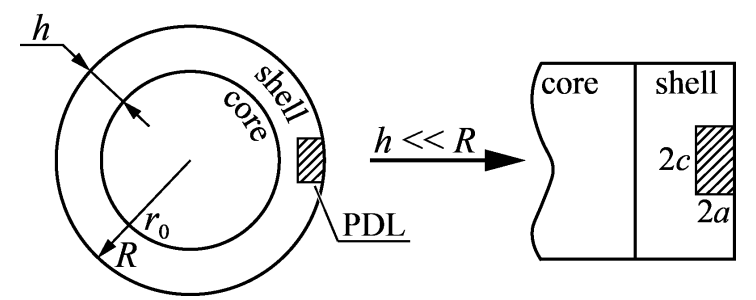

Fig. 14. Schematic representation of a core-shell NW cross section with a rectangular PDL $2 a \times 2 c$ and a planar model of the NW in the special case of a thin shell.

tions for the appearance of misfit PDLs with experimental observations was done within simpler approximate models for InAs-Al $\operatorname{In}_{1-x}$ As (with $x=0.20(f=1.3 \%), 0.33$ (2.5\%), and $0.53(32.6 \%)$ ) [37] and some other (GaAsGaP, GaSb-InSb, InAs-GaAs, InAs-InP, and InP-GaAs) [44] core-shell NWs. In general, the theoretical results occurred consistent with experimental data, although one should take into account that this approach gives only necessary but not sufficient conditions for the onset of misfit stress relaxation. Indeed, the general lack of the first group models is that they do not describe in detail neither the mechanism of misfit defect generation nor the energy barriers which must be overcome by the system to pass to a new partly relaxed state. This lack is deleted by using the second-group models that naturally include both these factors. Also, the height of the energy barrier seems to be more reliable criterion for the onset of misfit strain relaxation than the critical parameters calculated within the first-group models.

\section{ENERGY-BARRIER APPROACH FOR PREDICTING MD GENERATION IN CORE-SHELL NWs}

\subsection{Generation of small rectangular PDLs on either the interface or free surface of NWs}

To the best of our knowledge, one of the first known studies of the relaxation process in core-shell NWs related to the second group is works $[46,48]$. Therein, NWs were assumed to be elastically isotropic and homogeneous and the shell thickness $h$ was assumed to be much smaller than the NW outer radius $R$. Under these conditions, the authors suggested some models when the misfit strain relaxation process starts by the mechanisms of generation of small rectangular PDLs at either the core-shell interface or shell free surface with subsequent extension of the PDLs into the core or the shell. The limitation that $h<R$ allowed the authors to use the approximation of a PDL near a flat free surface (see Fig.
14) in calculation of the PDL strain energy. As a result, it was showed that the PDLs of the shape elongated along the interface are the most preferable and the free surface is the most favorable place for their generation. A detailed description of these models is given in work [48]. Here we just trace some principle stages of their examination.

Taking into account the foregoing assumptions, the authors of $[46,48]$ used the well-known solution for the strain energy $W^{d}$ of a rectangular PDL that lies in a plane perpendicular to the flat free surface of an elastic medium [107]:

$$
W^{d}=\frac{D b^{2} L}{2},
$$

where $b$ is the Burgers vector magnitude of the PDL and $L$ is the effective length which reads

$$
\begin{aligned}
& L=S_{1}+2 S_{2}+S_{3}+[3-4 v(3-2 v)] S_{4} \\
& +2 \frac{1-2 v\left(6-11 v+8 v^{3}\right)}{(1-2 v)^{2}} S_{5} \\
& -\frac{129-2 v\{234-v[245-4 v(5+16 v)]\}}{3(1-2 v)^{2}} S_{6},
\end{aligned}
$$

with the following denotations in the case of PDL generation from the NW free surface:

$$
\begin{aligned}
& S_{1}=c\left(2 \ln \frac{K_{3}+c}{K_{3}-c}-\ln \frac{\sqrt{c^{2}+\left(r_{c} / 2\right)^{2}}+c}{\sqrt{c^{2}+\left(r_{c} / 2\right)^{2}}-c}\right. \\
& \left.-\ln \frac{K_{2}+c}{K_{2}-c}\right), \\
& S_{2}=2 c \ln \frac{4 c}{r_{0}}+2 a \ln \frac{4 a}{r_{0}}-c \ln \frac{K_{3}+c}{K_{3}-c} \\
& -a \ln \frac{K_{3}+a}{K_{3}-a}-4\left(c+a-K_{3}\right), \\
& S_{3}=\frac{8(1-v)(1-2 v) a^{3}}{c^{2}}-\frac{2\left(c^{2}+a^{2}\right) K_{3}}{c^{2}}+3 a, \\
& S_{4}=\frac{2 a^{2} K_{3}-4 a^{2} K_{2}}{3 c^{2}}, S_{5}=2 a \ln \frac{a+K_{3}}{a} \\
& -2 a \ln \frac{2 a+K_{2}}{2 a}, S_{6}=2 K_{3}-K_{1}-K_{2}, \\
& K_{1}^{2}=c^{2}, K_{2}^{2}=c^{2}+2 a^{2}, K_{3}^{2}=c^{2}+a^{2},
\end{aligned}
$$

$a$ and $c$ are the dimensions of the PDL (see Fig. 14).

The core energy $W^{c}$ of the PDL was estimated with usual approximation as follows: 

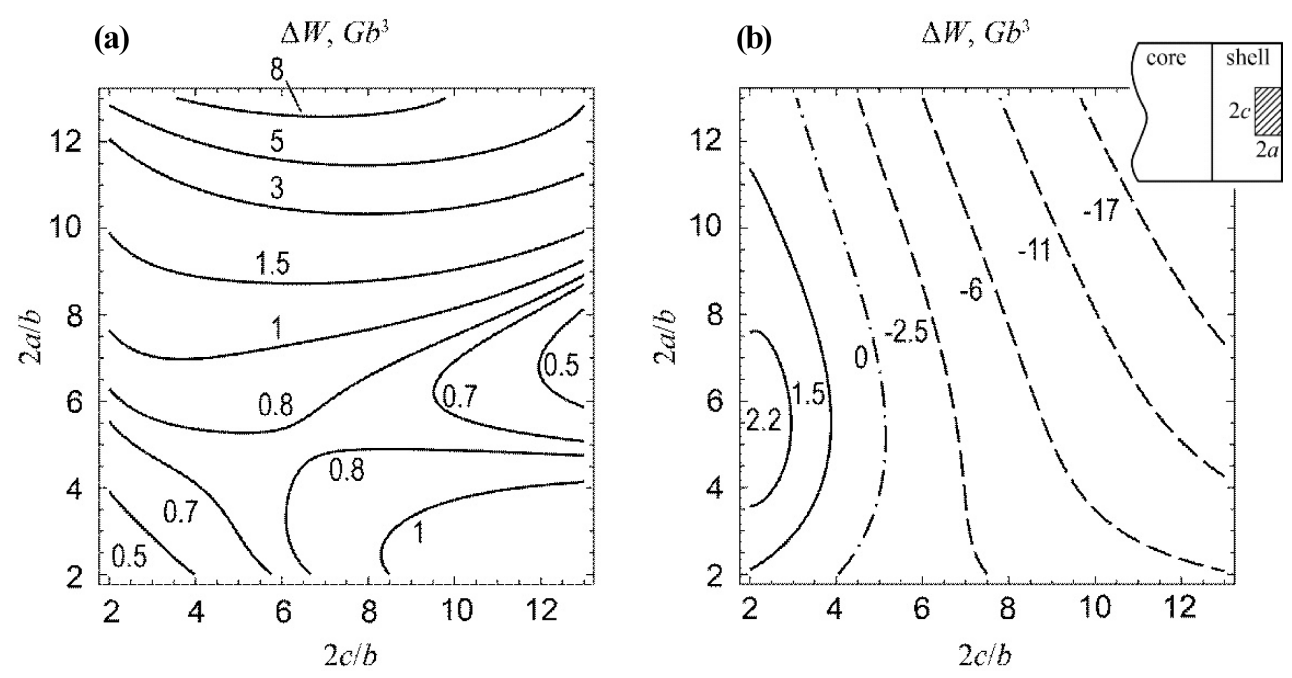

Fig. 15. The maps of the energy change $\Delta W$ in the space of normalized sizes of the PDL $(2 a / b, 2 c / b)$ for (a) Si-Ge and (b) InAs-ZnS core-shell NWs at $R=100 \mathrm{~nm}$ and $h=5 \mathrm{~nm}$. The PDL is generated from the NW free surface, see inset in (b). The energy values are given in units of $G b^{3}$.

$W^{c}=4\left(a+\frac{c}{2}\right) \frac{D b^{2}}{2}$.

The interaction energy $W^{\text {int }}$ was calculated as the work spent to nucleate the PDL in the misfit stress field in the shell of NW:

$W^{i n t}=-8 a b c G \frac{1+v}{1-v} f\left(\frac{r_{0}}{R}\right)^{2}$.

Fig. 15 shows the energy maps in the space of the loop normalized sizes for a PDL that generated from the free surface in Si-Ge (Fig. 15a) and InAs-ZnS (Fig. 15b) core-shell NWs. As is seen, the generation of the PDL on the free surface of the Ge shell in the Si-Ge NWs is energetically unfavorable (Fig. 15a), whereas the generation of the PDL in the $\mathrm{ZnS}$ shell of the InAs-ZnS NWs is energetically favorable only for the PDLs elongated along the free surface (when $2 c>6 b$, Fig. 15b). PDLs of other configurations must overcome large energy barriers that prevent their nucleation.

Thus, following [26,46,48] and Eqs. (18)-(20), one can assume that initial strained state can relax through generation of PDLs as shown in Fig. 16. The PDLs first nucleate on the free shell surface and further extend into the shell. Nucleating PDLs have a rectangular shape which further transforms in such a way that PDLs could extend along the interface and form a growing arc of MD at the interface. As a result, closed circular misfit PDLs form around the cores in the cross section of NWs at the final stage of the relaxation process [48].

It is worth noting that similar theoretical models were suggested to describe the critical conditions for the generation of either closed circular [47,85,108-111] or initial small rectangular [46-48,54,112] PDLs in spherically symmetric core-shell nanoparticles. In doing so, Gutkin et al. [47] noticed that the formation of rectangular PDLs does not necessarily leads to the formation of circular PDLs and, at the same time, the formation of circular PDLs does not necessarily occurs by the mechanism of initially rectangular PDL expansion and closure. Therefore, the formation of both can be considered to a first approximation as two independent stress relaxation ways. At the same time, if both ways could be applied to identical nanoparticles, it is reasonable to assume that they are interrelated according to the schematics shown in Fig. 16. Based on these assumptions, Gutkin et al. [47] compared the critical conditions of nucleation of such PDLs. They showed that either a coherent (dislocationfree) state of the nanoparticle or its relaxed state with a circular PDL at the interface is favorable in the case of a relatively small lattice misfit between the core and shell materials. For large misfits, the coherent state is unfavorable. In this case, as the shell thickness in-

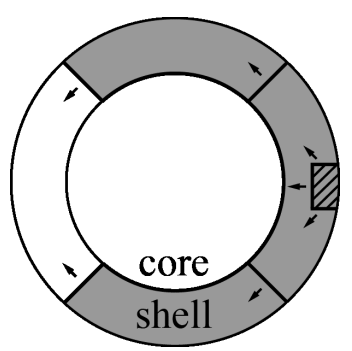

Fig. 16. Initial and intermediate stages of the generation of a PDL from the free surface of the shell to its bulk. The arrows show the directions of the loop expansion. The Figure is a sketch of Fig. 2b from the work [48], all major designations are saved. 


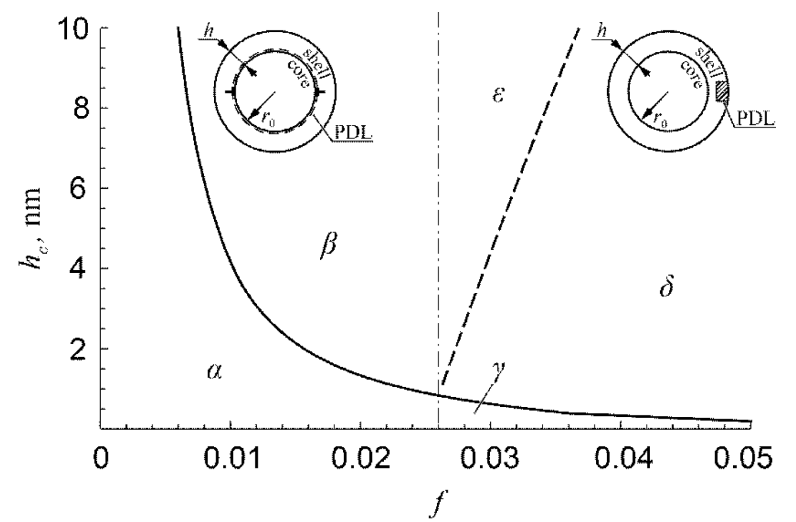

Fig. 17. Dependences of the critical shell thickness $h_{c}$ on the misfit parameter $f$ at $r_{0}=48 \mathrm{~nm}$ in the cases of nucleation of circular (solid curve) and rectangular (dashed curve) PDLs. The radius of a circular PDL equals to the core radius $r_{0}$, the sizes of the rectangular PDL are $a / c=0.2$ and $c / b=10, b=0.3 \mathrm{~nm}$.

creases, it can be expected that, first, rectangular PDLs will appear, then circular PDLs will be formed while retaining rectangular PDLs, and then rectangular PDLs will gradually grow and transform to circular PDLs.

Following Gutkin et al. [47], similar comparison of relaxation processes (nucleation of circular or rectangular PDLs) can be done for core-shell NWs as well, by using the dependences of the critical shell thickness $h_{c}$ on the misfit parameter $f$. Fig. 17 shows these dependences for circular (solid curves) and rectangular (dashed curves) PDLs in NWs with the core radius $r_{0}=$ $48 \mathrm{~nm}$. The circular PDL nucleation is energetically favorable if $f$ and $h$ fall within the range over the corresponding solid curve $h_{c}^{\text {circ }}(f)$, while the rectangular PDL nucleation is favorable, if these parameters fall within the range under the corresponding dashed curve $h_{c}^{r e c}(f)$. It should be noted that, in the case of rectangular PDLs, the described dependence shows the maximum shell thickness for the presence of PDLs. Thus, in this case, the critical thickness $h$ has the opposite meaning in comparison with the traditional definition that shows the value of the film (shell in our case) thickness above which the nucleation of MDs becomes energetically favorable, which is also true for circular loops.

Fig. 17 contains five areas corresponding to different states of defect structure in the core-shell NW. The $\alpha$-area is the area of the coherent (MD free) NW state which is implemented at relatively small misfit values $(f<0.026)$. Circular PDLs can appear in the region of $\beta$-area, where nevertheless rectangular PDLs cannot nucleate. This means that the formation of circular PDLs from initial rectangular PDLs (Fig. 16) is inapplicable there. In the $\gamma$-area, it is possible for rectangular PDLs to nucleate, nevertheless, circular PDLs cannot be formed.
In the $\delta$-area, both rectangular and circular PDLs can appear. This means that the action of the mechanism of rectangular PDL growth and their transformation to circular PDLs (Fig. 16) can be expected in this area. The nucleation of new rectangular PDLs terminates at the upper bound of the $\delta$-area drawn by the curve $h_{c}^{\text {rec }}(f)$. However, rectangular PDLs which are formed for that moment, can continue to expand and transform to circular PDLs. Finally, in the $\varepsilon$-area, the transformation of rectangular PDLs to circular PDLs should gradually terminate, and new rectangular PDLs should not appear, while circular PDLs can continue to nucleate as in the neighboring $\beta$-area.

It should be noted that to date there is a number of works considering the initial process of relaxation as a result of the nucleation of rectangular PDLs in other various nanostructures, such as bi- and tri-nanolayers, hollow cylindrical core-shell NWs and solid core-shell NWs with hexagonal, squared and triangular cross sections of cores $[46,48,54,58,60]$. In this respect, one can compare the stability of such nanostructures to the generation of small rectangular PDLs from the free surface (similar to the PDL shown in Fig. 14). Note that the core and strain energies $\left(W^{c}\right.$ and $W^{d}$ ) of the PDLs were taken equal for all nanostructures, see Eqs. (18) and (19), respectively. Thus, it was necessary to calculate only the interaction energies between the PDLs and the misfit stresses in the corresponding nanostructure. For example, some of them read in

(i) bi-nanolayer

$$
\begin{aligned}
& W^{i n t}=-8 a b c G \frac{1+v}{1-v} f\left[Q_{2}+k(h-a)\right], \\
& Q_{2}=-f(H-h) / H+k(H-2 h) / 2, \\
& k=6 f h(H-h) / H^{3},
\end{aligned}
$$

where $H$ is the total thickness of a bi-nanolayer and $h$ is the film thickness in the bi-nanolayer;

(ii) tri-nanolayer

$W^{i n t}=-8 a b c G \frac{1+v}{1-v} f\left(1-\frac{2 h}{H^{*}}\right)$,

where $H^{*}$ is the total thickness of a tri-nanolayer;

(iii) core-shell nanoparticle

$$
\begin{aligned}
& W^{i n t}=-\frac{4 G(1+v) f b}{3(1-v)} r_{0}^{3} \\
& \times\left(\frac{4 a c}{R^{3}}-\frac{\sqrt{c^{2}+R^{2}}}{c R}+\frac{\sqrt{c^{2}+(R-2 a)^{2}}}{c(R-2 a)}\right),
\end{aligned}
$$


where $r_{0}$ is the core radius and $R$ is the nanoparticle radius;

(iv) nanowire with hexagonal core

$$
\begin{aligned}
& W^{i n t}=-b \int_{-a}^{a} \mathrm{~d} y \int_{-c}^{c} \sigma_{z z}^{h e x} \mathrm{~d} x, \\
& \sigma_{z z}^{h e x}=2 C\left(\left[{ }^{\infty} \Psi_{z z}^{h x}+{ }^{*} \Psi_{z z}^{h x}\right]_{k=\sqrt{3} / 3, c=-R_{0}}^{k=-\sqrt{3} / 3, c=R_{0}}\right. \\
& { }^{\infty} \Psi_{z z}^{h x}=-\frac{\pi}{2} \operatorname{sgn} \frac{y-y_{0}}{x-k y-c}, \\
& { }_{y_{0}=0}^{*} \Psi_{z z}^{h x}=-\cos \psi\left[q^{2} \cos (2 \theta+\psi)+\frac{4}{p^{2}}\left(\sin (2 \varphi+\psi) \ln \sqrt{p^{2} q^{2}-2 p q \cos (\varphi-\theta)+1}\right.\right. \\
& \left.\left.+\cos (2 \varphi+\psi) \tan ^{-1} \frac{p q \sin (\varphi-\theta)}{1-p q \cos (\varphi-\theta)}\right)\right]
\end{aligned}
$$

where $p=r / R, q=\rho / R, \rho=\sqrt{x_{0}^{2}+y_{0}^{2}}, \theta=\tan ^{-1}\left(y_{0} / x_{0}\right), \psi=\tan ^{-1} k,\left(x_{0}, y_{0}\right)$ and $(\rho, \theta)$ are the Cartesian and polar coordinates, respectively, of a hexagonal corner, $r$ and $\varphi$ are the dimensions of global polar coordinate system of the NW;

(v) nanowire with squared core

$$
\begin{aligned}
& W^{i n t}=-b \int_{-a}^{a} \mathrm{~d} y \int_{-c}^{c} \sigma_{z z}^{s q} \mathrm{~d} x
\end{aligned}
$$

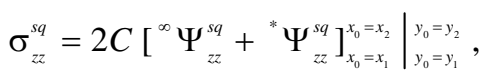

$$
\begin{aligned}
& { }^{\infty} \Psi_{z z}^{s q}=-\frac{\pi}{2} \operatorname{sgn} \frac{y-y_{0}}{x-x_{0}}, \\
& { }^{*} \Psi_{z z}^{s q}=-q^{2} \sin 2 \theta+\frac{4}{p^{2}}\left[\sin 2 \varphi \ln \sqrt{p^{2} q^{2}-2 p q \cos (\varphi-\theta)+1}-\cos 2 \varphi \tan ^{-1} \frac{p q \sin (\varphi-\theta)}{1-p q \cos (\varphi-\theta)}\right] .
\end{aligned}
$$

(vi) nanowire with triangular core

$$
\begin{aligned}
& W^{i n t}=-b \int_{-a}^{a} \mathrm{~d} y \int_{-c}^{c} \sigma_{z z}^{t r} \mathrm{~d} x, \\
& \sigma_{z z}^{t r}=2 C\left[{ }^{\infty} \Psi_{z z}^{t r}+{ }^{*} \Psi_{z z}^{t r}\right]_{k=\sqrt{3} / 3, c=-\sqrt{3} R_{0} / 3}^{k=-\sqrt{3} / 3, c=\sqrt{3} R_{0} / 3} \begin{array}{l}
y_{0}=R_{0} \\
y_{0}=-R_{0} / 2
\end{array}, \\
& { }^{\infty} \Psi_{z z}^{t r}=-\frac{\pi}{2} \operatorname{sgn} \frac{y-y_{0}}{x-k y-c}, \\
& { }^{*} \Psi_{z z}^{t r}=-\cos \psi\left[q^{2} \cos (2 \theta+\psi)+\frac{4}{p^{2}}\left(\sin (2 \varphi+\psi) \ln \sqrt{p^{2} q^{2}-2 p q \cos (\varphi-\theta)+1}\right.\right. \\
& \left.\left.+\cos (2 \varphi+\psi) \tan ^{-1} \frac{p q \sin (\varphi-\theta)}{1-p q \cos (\varphi-\theta)}\right)\right] .
\end{aligned}
$$

In the cases of NWs with faceted cores, the interaction energies $W^{\text {int }}$ were calculated numerically within the Wolfram Mathematica software.

In order to compare the critical relaxation conditions in composite nanostructures, it is more convenient to use the dependences of the critical shell (film for flat heterostructures) thickness $h_{c}$ on the misfit parameter $f$ (see Fig. 18). 

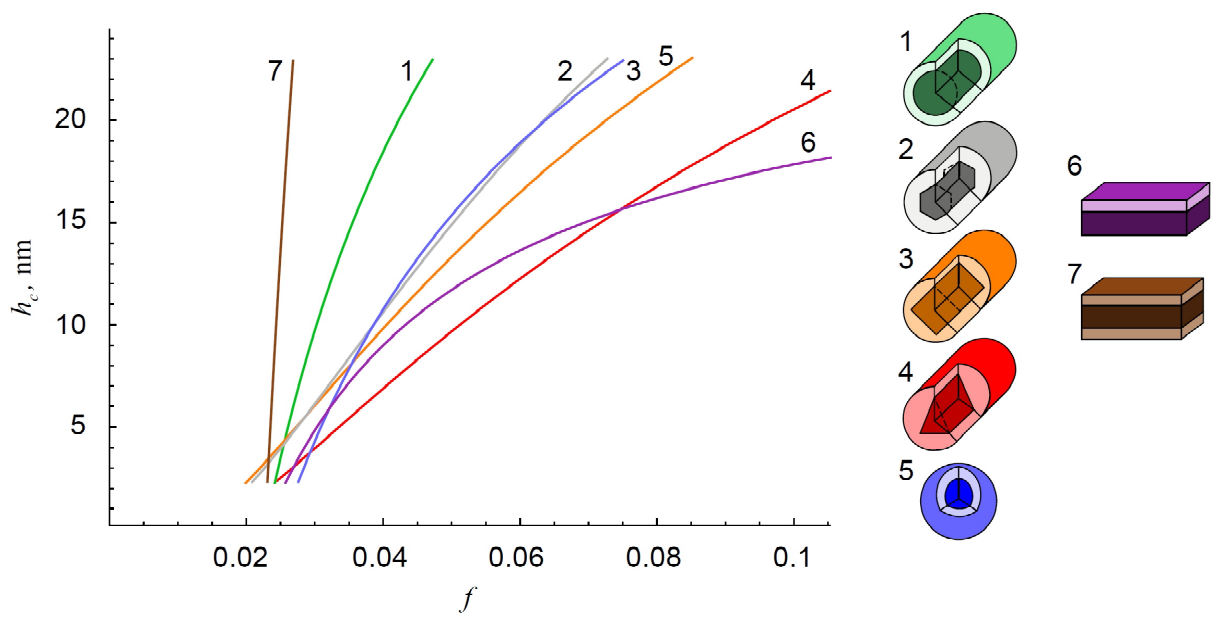

Fig. 18. Dependences of the critical shell (film) thickness $h_{c}$ on the misfit parameter $f$ for various nanostructures in which PDLs are generated from the free surface. The outer radius of NWs and nanoparticle is $R=75 \mathrm{~nm}$, the full thickness of bi-nanolayer is $H=R=75 \mathrm{~nm}$, the full thickness of tri-nanolayer is $H^{*}=2 H=2 R=150 \mathrm{~nm}$, the PDL sizes are $a / c=0.2$ and $c / b=10, b=0.3$. In the case of NW with polyhedral cores, the shell thickness was taken as the distance between the core corner and the free cylindrical surface.

The generation of PDLs in a nanostructure is energetically favorable when the values of $h$ and $f$ fall into the area under the curve (Fig. 18). Therefore, the most stable nanostructure in this sense is either NW with triangular core cross section, in the range of $f<0.075$, or bi-nanolayer, in the range of $f>0.075$, as it is characterized by the smallest area under the corresponding curve. On the other hand, the tri-nanolayer is the least resistant to the PDL formation. The symmetry of the trinanolayer structure excludes its bending and, as a result, the films covering the substrate suffer larger misfit stresses than in the case of bi-nanolayer with the film of the same thickness. The stability of nanoparticles is close to the stability of a NW with hexagonal core cross section. NWs with circular core cross section are the least stable among core-shell NWs against nucleation of PDLs from the free surface.

\subsection{Generation of straight MDs near the edges of prismatic cores}

Besides generation of PDLs, various mechanisms of nucleation of straight edge dislocations, leading to the formation of straight MDs at the core-shell interface, were considered within the energy-barrier approach as well. In doing so, Smirnov et al. [61] recently analyzed the energy barriers for generation of partial and perfect straight edge dislocations and their dipoles at the interface in core-shell NWs with faceted cores (Fig. 19). The core had the shape of a long parallelepiped of a square cross-section and was placed symmetrically with respect to the cylindrical shell surface. For the sake of definiteness, the authors did their calculations for model metal gold/palladium NWs, however, similar calculations could be performed for semiconductor NWs with using the appropriate parameters of the material.

As discussed earlier, generation of MDs leading to the misfit stress relaxation is energetically favorable if the corresponding change in the total energy of the system $\Delta W$ is negative. According to the type of the defect structure, the energy change $\Delta W$ is given by one of the following four equations.

For a partial MD:

$$
\begin{aligned}
& \Delta W=W^{d}\left(b_{p a r}\right)+W^{c}\left(b_{p a r}\right)+W^{i n t}\left(b_{p a r}\right) \\
& +W^{s s}\left(b_{p a r}\right)+W^{\gamma}\left(b_{p a r}\right) .
\end{aligned}
$$

For a perfect MD:
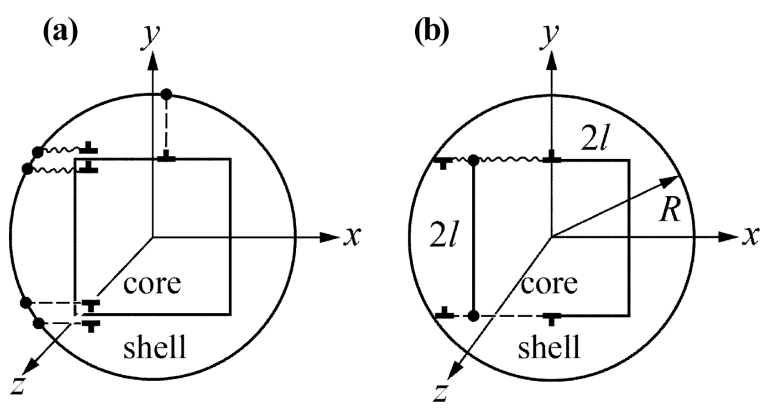

Fig. 19. Schematic representation of a core-shell NW cross section with faceted core. (a) Glide (on the left) or climb (on the right) of partial (top left) and perfect (bottom left and top right) MDs from the shell free surface. (b) Nucleation of dipoles of partial (top) and perfect (bottom) MDs at the edges of the core. The core cross section is a square with the side $2 l$. Figure is a sketch of Fig. 1 from the work [61], all major designations are saved. 


$$
\Delta W=W^{d}\left(b_{p e r}\right)+W^{c}\left(b_{p e r}\right)+W^{i n t}\left(b_{p e r}\right)+W^{s s}\left(b_{p e r}\right) .
$$

For a dipole of partial MDs (DMDs):

$$
\Delta W=W_{D M D s}^{d}\left(b_{p a r}\right)+2 W^{c}\left(b_{p a r}\right)+W_{D M D s}^{\text {int }}\left(b_{p a r}\right)+W_{D M D s}^{\gamma}\left(b_{p a r}\right) .
$$

For a dipole of perfect MDs:

$$
\Delta W=W_{D M D s}^{d}\left(b_{p e r}\right)+2 W^{c}\left(b_{p e r}\right)+W_{D M D s}^{i n t}\left(b_{p e r}\right) .
$$

Here $W^{s s}$ is the energy of step formation during the generation of the MD by glide from the free surface, $W^{\prime}$ is the stacking fault energy, $b_{p a r}\left(b_{p e r}\right)$ is the Burgers vector magnitude of a partial (perfect) dislocation.

The strain energy $W^{d}$ of an individual edge dislocation (Fig. 19a) in a NW reads [113]:

$$
\begin{aligned}
& W^{d}\left(b^{\prime}\right)=\frac{D b^{\prime 2}}{8} G^{*}, \\
& G^{*}=\left(M_{+}-1\right)\left(1-2 \tilde{y}_{0}^{2}\left(M_{+}+1\right)\right)+\left(M_{-}-1\right)\left(1-2 \tilde{y}_{0}^{2}\left(M_{-}+1\right)\right)-\ln \left(M_{+} M_{-}\right), \\
& M_{ \pm}=\frac{\tilde{r}_{0}^{2}}{\tilde{y}_{0}^{4}+\left(\tilde{x}_{0}^{2}+\left(\tilde{x}_{0} \pm \tilde{r}_{0}\right)^{2}-2\right) \tilde{y}_{0}^{2}+\left(\tilde{x}_{0}^{2} \pm \tilde{r}_{0} \tilde{x}_{0}-1\right)^{2}}, \quad \tilde{x}_{0}=\frac{x_{0}}{R}, \quad \tilde{y}_{0}=\frac{y_{0}}{R}, \tilde{r}_{0}=\frac{b^{\prime}}{R},
\end{aligned}
$$

where $x_{0}$ and $y_{0}$ are the Cartesian coordinates of the dislocation, $b^{\prime}=b_{p a r}$ for the partial MD and $b^{\prime}=b_{p e r}$ for the perfect MD.

Using the approach suggested in [113], Smirnov et al. [61] calculated the strain energy of an edge dislocation dipole (Fig. 19b) as follows:

$$
\begin{aligned}
& W_{D M D s}^{d}=\frac{1}{4} G^{*} D b^{\prime 2}, \\
& G^{*}=\left(M_{1}-1\right)\left(1-2 y_{0}^{2} \frac{M_{1}+1}{R^{2}}\right)-\left(M_{2}-1\right)\left(1-2 y_{0}^{2} \frac{M_{2}+1}{R^{2}}\right) \\
& +\left(M_{4}-1\right)\left(1-2 y_{0}^{2} \frac{M_{4}+1}{R^{2}}\right)-\left(M_{3}-1\right)\left(1-2 y_{0}^{2} \frac{M_{3}+1}{R^{2}}\right)+\ln \frac{M_{2} M_{3}}{M_{1} M_{4}}, \\
& M_{1}=\frac{R^{2} b^{\prime 2}}{R^{4}-2 R^{2}\left(x_{1}\left(x_{1}+b^{\prime}\right)+y_{0}^{2}\right)+\left(\left(x_{1}+b^{\prime}\right)^{2}+y_{0}^{2}\right)\left(x_{1}^{2}+y_{0}^{2}\right)}, \\
& M_{2}=\frac{R^{2}\left(x_{2}-x_{1}-b^{\prime}\right)^{2}}{R^{4}-2 R^{2}\left(x_{1}\left(x_{2}-b^{\prime}\right)+y_{0}^{2}\right)+\left(\left(x_{2}-b^{\prime}\right)^{2}+y_{0}^{2}\right)\left(x_{1}^{2}+y_{0}^{2}\right)}, \\
& M_{3}=\frac{R^{2}\left(x_{1}-x_{2}+b^{\prime}\right)^{2}}{R^{4}-2 R^{2}\left(x_{2}\left(x_{1}+b^{\prime}\right)+y_{0}^{2}\right)+\left(\left(x_{1}+b^{\prime}\right)^{2}+y_{0}^{2}\right)\left(x_{2}^{2}+y_{0}^{2}\right)}, \\
& M_{4}=\frac{R^{2} b^{\prime 2}}{R^{4}-2 R^{2}\left(x_{2}\left(x_{2}-b^{\prime}\right)+y_{0}^{2}\right)+\left(\left(x_{2}-b^{\prime}\right)^{2}+y_{0}^{2}\right)\left(x_{2}^{2}+y_{0}^{2}\right)},
\end{aligned}
$$

where $x_{1}, x_{2}$ and $y_{0}$ are Cartesian coordinates of the MDs.

The MD core energy $W^{c}$ was estimated by standard Eq. (3).

The interaction energy $W^{\text {int }}$ for a gliding MD (Fig. 19a) was calculated as the work spent to generate an individual MD in the NW misfit stress $\sigma_{x y}$ that is given by the sum $\sigma_{x y}=\sigma_{x y}^{\infty}+\sigma_{x y}^{*}$ (the details on calculating the stress field in a NW with parallelepipedal core are given in [61]), as: 


$$
\begin{aligned}
& W^{i n t}\left(b^{\prime}\right)=-\left.b^{\prime} \int_{-\sqrt{R^{2}-l^{2}}}^{x_{0}} \sigma_{x y}^{\infty}\right|_{y=y_{0}} \mathrm{~d} x-\left.b^{\prime} \int_{-\sqrt{R^{2}-l^{2}}}^{x_{0}} \sigma_{x y}^{*}\right|_{y=y_{0}} \mathrm{~d} x, \\
& \sigma_{x y}^{\infty}=C \ln \frac{\left[(x-l)^{2}+(y-l)^{2}\right]\left[(x+l)^{2}+(y+l)^{2}\right]}{\left[(x-l)^{2}+(y+l)^{2}\right]\left[(x+l)^{2}+(y-l)^{2}\right]}, \\
& \sigma_{x y}^{*}=\left(\sigma_{r r}^{*}-\sigma_{\theta \theta}^{*}\right) \frac{x y}{x^{2}+y^{2}}+\sigma_{r \theta}^{*} \frac{x^{2}-y^{2}}{x^{2}+y^{2}}, \\
& \sigma_{r r}^{*}=4 C \rho^{2}\left[1-\sum_{n=1}^{+\infty} \frac{(-1)^{n}}{2 n+1}\left(2(2 n-1)-(4 n-1) \frac{R^{2}}{r^{2}}\right)\left(\frac{\rho r}{R}\right)^{4 n} \cos 4 n \theta\right] \\
& \sigma_{r \theta}^{*}=4 C \rho^{2} \sum_{n=1}^{+\infty} \frac{(-1)^{n}}{2 n+1}\left(4 n-(4 n-1) \frac{R^{2}}{r^{2}}\right)\left(\frac{\rho r}{R}\right)^{4 n} \sin 4 n \theta, \\
& \sigma_{\theta \theta}^{*}=4 C \rho^{2}\left[1+\sum_{n=1}^{+\infty} \frac{(-1)^{n}}{2 n+1}\left(2(2 n+1)-(4 n-1) \frac{R^{2}}{r^{2}}\right)\left(\frac{\rho r}{R}\right)^{4 n} \cos 4 n \theta\right], \\
& \sigma_{z z}^{*}=16 C \rho^{2} \sum_{n=1}^{+\infty} \frac{(-1)^{n}}{2 n+4}\left(\frac{\rho r}{R}\right)^{4 n} \cos 4 n \theta,
\end{aligned}
$$

where $C=G f(1+v) /[2 \pi(1-v)], \rho=\sqrt{2} l / R,\left(x_{0}, y_{0}\right)$ are the Cartesian coordinates of the MD, $\theta=\tan ^{-1}(y / x)$, $r=\sqrt{x^{2}+y^{2}},(x, y)$ and $(r, \theta)$ are the Cartesian and polar coordinate systems respectively.

In the case of individual MD generation by climbing (Fig. 19a), the interaction energy $W^{\text {int }}$ was determined by the following expression:

$$
\begin{aligned}
& W^{i n t}\left(b^{\prime}\right)=-\left.b_{p e r} \int_{y_{0}}^{R}\left(\sigma_{x x}^{\infty}-\sigma^{\infty} / 3\right)\right|_{x=0} \mathrm{~d} y-\left.b_{p e r} \int_{y_{0}}^{R}\left(\sigma_{x x}^{*}-\sigma^{*} / 3\right)\right|_{x=0} \mathrm{~d} y, \\
& \sigma^{\infty}=\sigma_{x x}^{\infty}+\sigma_{y y}^{\infty}+\sigma_{z z}^{\infty}, \sigma^{*}=\sigma_{x x}^{*}+\sigma_{y y}^{*}+\sigma_{z z}^{*}, \\
& \sigma_{x x}^{\infty}=-2 C\left(\tan ^{-1} \frac{x-l}{y-l}-\tan ^{-1} \frac{x-l}{y+l}-\tan ^{-1} \frac{x+l}{y-l}+\tan ^{-1} \frac{x+l}{y+l}\right), \\
& \sigma_{y y}^{\infty}=-2 C\left(\tan ^{-1} \frac{y-l}{x-l}-\tan ^{-1} \frac{y-l}{x+l}-\tan ^{-1} \frac{y+l}{x-l}+\tan ^{-1} \frac{y+l}{x+l}\right), \\
& \sigma_{z z}^{\infty}=-\pi C\left(\operatorname{sgn} \frac{x-l}{y-l}-\operatorname{sgn} \frac{x-l}{y+l}-\operatorname{sgn} \frac{x+l}{y-l}+\operatorname{sgn} \frac{x+l}{y+l}\right), \\
& \sigma_{x x}^{*}=\sigma_{r r}^{*} \frac{x^{2}}{x^{2}+y^{2}}+\sigma_{\theta \theta}^{*} \frac{y^{2}}{x^{2}+y^{2}}-2 \sigma_{r \theta}^{*} \frac{x y}{x^{2}+y^{2}} \text {, } \\
& \sigma_{y y}^{*}=\sigma_{r r}^{*} \frac{y^{2}}{x^{2}+y^{2}}+\sigma_{\theta \theta}^{*} \frac{x^{2}}{x^{2}+y^{2}}+2 \sigma_{r \theta}^{*} \frac{x y}{x^{2}+y^{2}} \text {. }
\end{aligned}
$$

The interaction energy $W_{D M D s}^{\text {int }}$ was calculated as the work spent to generate a dipole of MDs (Fig. 19b) in the misfit stress field $\sigma_{x y}=\sigma_{x y}^{\infty}+\sigma_{x y}^{*}$ in the glide plane along the core/shell interface in the NW:

$$
W_{D M D s}^{i n t}=-b^{\prime} \int_{x_{1}}^{x_{2}} \sigma_{x y}\left(x, y=y_{0}\right) \mathrm{d} x .
$$

The stacking fault energy $W^{\gamma}$ for a partial MD was given by [113]: 


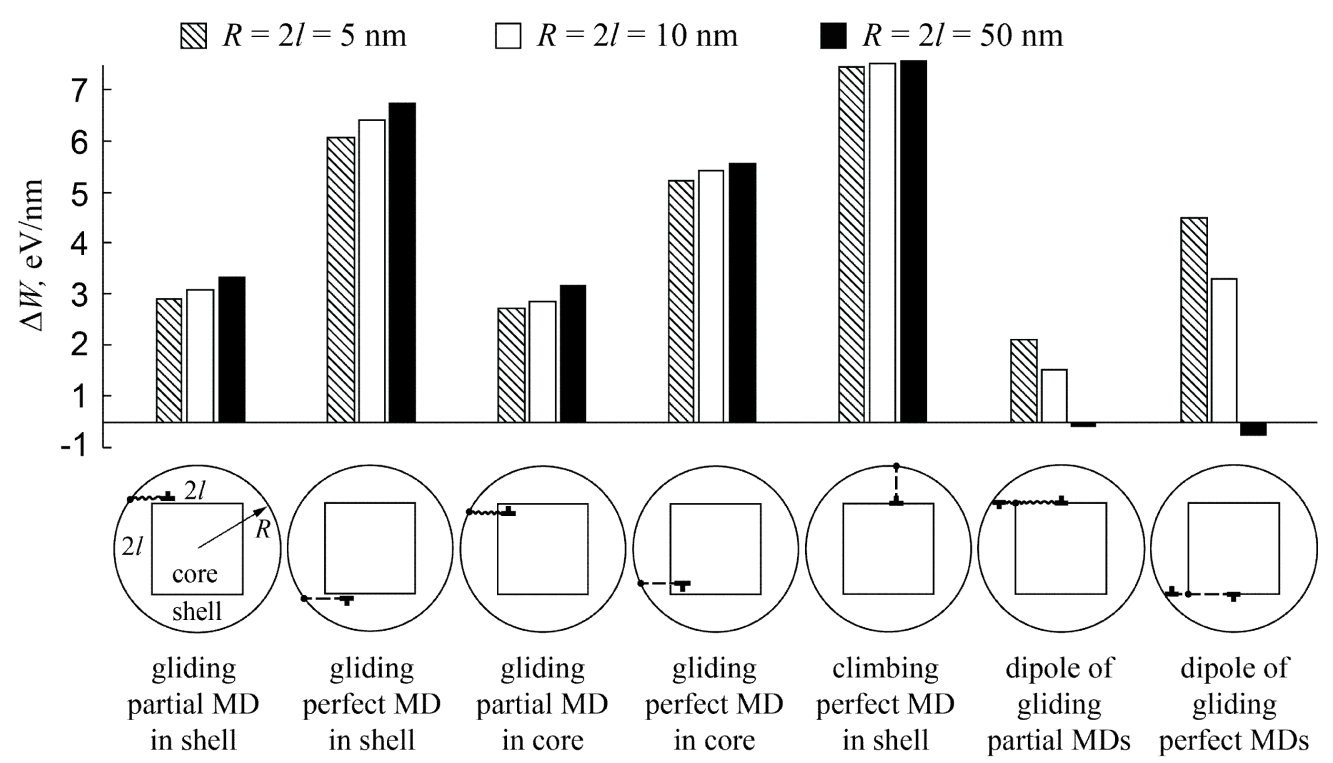

Fig. 20. The preference diagrams for various relaxation processes in core-shell NWs at $R=2 l$. The left scale shows the energy barrier value. The Figure is a sketch of Fig. 10a from the work [61], all major designations are saved.

$$
\begin{aligned}
& W^{\gamma}= \\
& \left\{\begin{array}{l}
\gamma_{1}\left(\sqrt{R^{2}-y_{0}^{2}}+x_{0}\right),-\sqrt{R^{2}-y_{0}^{2}}<x_{0}<-l \\
\gamma_{1}\left(\sqrt{R^{2}-y_{0}^{2}}-l\right)+\gamma_{2}\left(l+x_{0}\right),-l<x_{0}<0
\end{array}\right.
\end{aligned}
$$

In the case of a partial MDs dipole, it was:

$$
W_{D M D S}^{\gamma}=\gamma_{2}\left(x_{2}+l\right)-\gamma_{1}\left(x_{1}+l\right) \text {. }
$$

Here $\gamma_{1}$ is the specific energy of the stacking fault in the shell, and $\gamma_{2}$ is that in either the shell or core along the core-shell interface.

The energy of the surface step $W^{s s}$, which occurs due to the MD generation from the free surface of the shell, was approximated by the well-known formula:

$$
W^{s s}\left(b^{\prime}\right) \approx G b^{\prime 2} / 8 \approx D b^{\prime 2} / 2 \text {. }
$$

With Eqs. (27)-(38), Smirnov et al. [61] calculated energy barriers for each relaxation process in NWs shown in Fig. 19. In doing so, they used the following parameters in their calculations: $a_{\mathrm{Au}}=0.408 \mathrm{~nm}$ and $a_{\text {Pd:Au }=50: 50}=0.398 \mathrm{~nm}$; the Burgers vector magnitudes $b_{p a r}=a / \sqrt{6}$ and $b_{p e r}=a / \sqrt{2}$; the misfit value $f=0.046$; the Poisson ratios $v_{\mathrm{Au}}=0.4$ and $v_{\mathrm{Pd}: \mathrm{Au}=50: 50}=0.395$; the shear moduli $G_{\mathrm{Au}}=27 \mathrm{GPa}$ and $G_{\mathrm{Pd}: \mathrm{Au}=50: 50}=35.5 \mathrm{GPa}$; the stacking fault energies $\gamma_{\mathrm{Au}}=31 \mathrm{~mJ} / \mathrm{m}^{2}, \gamma_{\mathrm{Pd}: \mathrm{Au}=60: 40}=120$ $\mathrm{mJ} / \mathrm{m}^{2}, \gamma_{\mathrm{Pd}: \mathrm{Au}=50: 50}=108 \mathrm{~mJ} / \mathrm{m}^{2}, v_{\mathrm{Au}} \approx v_{\mathrm{Pd}: \mathrm{Au}} \approx \mathrm{v}, G_{\mathrm{Au}} \approx$ $G_{\mathrm{Pd}: \mathrm{Au}} \approx G$. Note that the authors took various values of the stacking fault energy, depending on the positions of those partial MDs which are generated in the shell only, $\left.\gamma_{1}\right|_{-\sqrt{R^{2}-l^{2}}<x_{0}<-l}=\gamma_{\text {Pd:Au }=60: 40}$ and $\left.\gamma_{2}\right|_{-l<x_{0}<0}=\gamma_{\text {Pd:Au }=50: 50}$, and those partial MDs which are generated in the shell and core: $\left.\gamma_{1}\right|_{-\sqrt{R^{2}-l^{2}<x_{0}<-l}}=\gamma_{\mathrm{Pd}: \mathrm{Au}=60: 40}$ and $\left.\gamma_{2}\right|_{-l<x_{0}<0}=\gamma_{\mathrm{Au}}$.

The comparison of these calculations is visualized through the preference diagrams shown in Fig. 20. As is seen, the energy barriers for generation of MD dipoles are always lower than those for generation of individual MDs of the same type (either partial or perfect). Therefore, the formation of MD dipoles from the core edge is more expectable than the generation of individual MDs from the free surface of the shell. Dipoles of partial MDs are more expectable in relatively thin NWs than dipoles of perfect MDs; however, in relatively thick nanowires, one should expect that the most energetically favorable defects are the dipoles of perfect MDs. It should be noted that this fact is also valid for individual MDs. At $R=2 l$, the generation of perfect MDs by climb from the free surface is the least favorable mechanism of relaxation.

\section{CONCLUSIONS}

In conclusion, we have reviewed some analytical models aimed at predicting the critical conditions for the generation of MDs and related dislocation structures in core-shell NWs. We have shown that most of analytical models describe the misfit relaxation process in coreshell NWs through the generation of various defects in their final configurations within the quasi-equilibrium energy approach. These models allow to define the critical parameters (mostly the critical sizes of NWs and critical value of the misfit parameter) at which the formation of the misfit defects becomes energetically favorable. The main results, achieved by this group of models, can be summarized as follows: 
- The most common final MD configurations in coreshell NWs, being under discussion, are straight MDs and closed misfit PDLs.

- The most universal critical parameter is the critical misfit value $f_{c}$ which is easily extracted from the energy balance due to linear contribution of the misfit parameter $f$ in the balance.

- For any values of the NW radius $R$ and the ratio $t=r_{0} / R$ of the inner and outer radii of the NW, one can find the minimum value $f_{c \text {,min }}$ of the critical misfit such as for $f<f_{c, \text { min }}$, MD generation is not energetically favorable; when $f>f_{c \text {, min }}$, there is an interval of $t$ values, $t_{c 1}<t<t_{c 2}$, where $t_{c i}=t_{c i}(f, R), i=1,2$, in which MD generation is energetically favorable; when $f \rightarrow f_{c, \text { min, }} t_{c 1} \rightarrow t_{c 2}$.

- The larger is the NW radius $R$, the smaller is the minimum critical misfit $f_{c, \text { min }}$.

- For a fixed value of $f$, one can find the minimum critical radius of the NW, $R=R_{c, \text { min }}$, such as for $R<R_{c, \text { min }}$, MD generation is not energetically favorable for any value of $t$.

- The larger is the misfit value $f$, the smaller is the minimum critical radius of the NW $R_{c \text {,min }}$.

- In the case of thin shell on massive core (the shell thickness $h<<R$ ), when $f>f_{c \text {, min }}$, the critical condition can be taken in the form of $h>h_{c}$, where the critical shell thickness has the same meaning as for flat film-substrate heterostructures; in this case, the larger are the NW radius $R$ and the misfit value $f$, the smaller is the critical shell thickness $h_{c}$.

- In the case of thick shell on thin core (the shell thickness $h \gg r_{0}$ ), when $f>f_{c \text {, min }}$, the critical condition can be taken in the form of $r_{0}>r_{0 c}$, where $r_{0 c}$ is the critical radius of the core; in this case, the larger are the NW radius $R$ and the misfit value $f$, the smaller is $r_{0 c}$.

- The difference in elastic constants of the core and shell materials, as well as the elastic constants of the shell free surface and the core-shell interface, and the surface and interface tensions can strongly influence the critical conditions of MD generation in core-shell NWs. In particular, the positive (negative) surface/interface elastic constants mostly make the generation of MDs easier (harder). On the contrary, the positive (negative) residual surface/interface tensions mostly make their generation harder (easier).

- In the case, when $f>f_{c \text {, min }}$ and $t_{c 1}<t<t_{c 2}$, the equilibrium density of misfit PDLs can be calculated; the results of corresponding calculations for InAs-GaAs coreshell NWs are in a good accordance with experimental data.

Another group of relaxation models for core-shell NWs considered the energy barriers for nucleation and evolution of the defect configuration that allowed to choose between different ways of misfit relaxation and to predict the final misfit defect structure in NWs. The following results on this direction were obtained:
- The analysis was done for the models dealing with nucleation of small rectangular PDLs and straight edge MDs on free surfaces of the shells and at core-shell interfaces of various shapes.

- Nucleation of small rectangular PDLs was investigated in the case of thin shell on massive core for different shapes of the core cross section: circular, triangular, square and hexagonal.

- The most probable scenario for PDL appearance in the core-shell NWs is the formation on the shell free surface of small rectangular PDLs extended along this surface in the cross sections of the NWs.

- The critical conditions for nucleation of small rectangular PDLs of fixed sizes can be formulated either as $f>f_{c}$ (for given values of $R$ and $h$ ) or $h<h_{c}^{*}$ (for given values of $R$ and $f$ ), where $h_{c}^{*}$ has the different meaning to that traditionally used in the case of flat film-substrate heterostructures: it is the maximal thickness of the shell at which the misfit strain/stress is sufficient for generation of PDLs.

- The larger are the NW radius $R$ and the misfit value $f$, the larger is the critical thickness $h_{c}{ }^{*}$.

- Nucleation of straight edge MDs was considered in the case of core-shell NWs with prismatic core of square cross section.

- Among some different mechanisms of misfit strain relaxation in such NWs, the nucleation of glide MD dipoles at the core edges was shown to be the most probable; in the exemplary case of Au-Pd core-shell NWs, the dipoles of partial MDs are the most expectable in the thinnest NWs of radius 5-10 nm, while dipoles of perfect MDs are the most expectable in the coarser NWs of radius $50 \mathrm{~nm}$.

- The generation of perfect MDs by climb from the free surface is the least favorable mechanism of relaxation in the core-shell NWs.

Finally, by analogy with the Matthews-Blakeslee critical film thickness approach for flat film/substrate heterostructures, most of the considered models suggest the dependences of the critical shell thickness on the parameters of the NW materials. Such dependences are convenient from the practical perspective, since they are able to reduce the number of expensive experiments aimed at the synthesizing of dislocation-free NWs which have a great impact on quantum communications in future networks. The predicted defect structure in coreshell NWs could be of interest to researchers dealing with elaboration and characterization of the NWs.

\section{ACKNOWLEDGMENT}

This work was supported by the Ministry of Science and Higher Education of the Russian Federation, research project no. 2019-1442. 


\section{REFERENCES}

[1] Y. Chong, A Brief Review on the Recent Progress of Superconducting Nanowire Single Photon Detectors, Prog. Supercond. Cryog., 2017, vol. 19, no. 4, pp. 22-25. https://doi.org/10.9714/ psac.2017.19.4.022

[2] J.-W. Min, D. Priante, M. Tangi, G. Liu, C.H. Kang, A. Prabaswara, C. Zhao, L. Al-Maghrabi, Y. Alaskar, A.M. Albadri, A.Y. Alyamani, T.K. Ng and B.S. Ooi, Unleashing the Potential of Molecular Beam Epitaxy Grown AlGaN-Based UltravioletSpectrum Nanowires Devices, J. Nanophotonics, 2018, vol. 12, no. 4, art. 043511. https://doi.org/ 10.1117/1.JNP.12.043511

[3] A. Sajid, M.J. Ford and J.R. Reimers, SinglePhoton Emitters in Hexagonal Boron Nitride: a Review of Progress, Reports Prog. Phys., 2020, vol. 83, no. 4, art. 044501. https://doi.org/10.1088/ 1361-6633/ab6310

[4] J.S. Marsland, On the Effect of Ionization Dead Spaces on Avalanche Multiplication and Noise for Uniform Electric Fields, J. Appl. Phys., 1990, vol. 67, no. 4, pp. 1929-1933. https://doi.org/ 10.1063/1.345596

[5] C. Hu, K.A. Anselm, B.G. Streetman and J.C. Campbell, Noise Characteristics of Thin Multiplication Region GaAs Avalanche Photodiodes, Appl. Phys. Lett., 1996, vol. 69, no. 24, pp. 3734-3736. https://doi.org/10.1063/1.117205

[6] K. Kim and K. Hess, Simulations of Electron Impact Ionization Rate in GaAs in Nonuniform Electric Fields, J. Appl. Phys., 1986, vol. 60, no. 7, pp. 2626-2629. https://doi.org/10.1063/1.337135

[7] G. Bulgarini, M.E. Reimer, M. Hocevar, E.P.A.M. Bakkers, L.P. Kouwenhoven and V. Zwiller, Avalanche Amplification of a Single Exciton in a Semiconductor Nanowire, Nat. Photonics, 2012, vol. 6, no. 7, pp. 455-458. https://doi.org/10.1038/ nphoton.2012.110

[8] A.C. Farrell, P. Senanayake, C.-H. Hung, G. El-Howayek, A. Rajagopal, M. Currie, M.M. Hayat and D.L. Huffaker, Plasmonic Field Confinement for Separate Absorptionmultiplication in InGaAs Nanopillar Avalanche Photodiodes, Sci. Rep., 2015, vol. 5, no. 1, art. 17580. https://doi.org/10.1038/srep17580

[9] V. Jain, M. Heurlin, E. Barrigon, L. Bosco, A. Nowzari, S. Shroff, V. Boix, M. Karimi, R.J. Jam, A. Berg, L. Samuelson, M.T. Borgström, F. Capasso and H. Pettersson, InP/InAsP Nanowire-Based Spatially Separate Absorption and Multiplication Avalanche Photodetectors,
ACS Photonics, 2017, vol. 4, no. 11, pp. 2693-2698. https://doi.org/10.1021/acsphotonics.7b00389

[10] A.C. Farrell, X. Meng, D. Ren, H. Kim, P. Senanayake, N.Y. Hsieh, Z. Rong, T.-Y. Chang, K.M. Azizur-Rahman and D.L. Huffaker, InGaAsGaAs Nanowire Avalanche Photodiodes Toward Single-Photon Detection in Free-Running Mode, Nano Lett., 2019, vol. 19, no. 1, pp. 582590. https://doi.org/10.1021/acs.nanolett.8b04643

[11] S. Giaremis, P. Komninou, I. Belabbas, J. Chen and J. Kioseoglou, Structural and electronic properties of a-edge dislocations along <1-100> in GaN, J. Appl. Phys., 2018, vol. 123, no. 24, art. 244301. https://doi.org/10.1063/ 1.5034198

[12] I. Goldthorpe, A.F. Marshall and P.C. McIntyre, Synthesis and Strain Relaxation of Ge-Core / SiShell Nanowire Arrays, Nano Lett., 2008, vol. 8, no. 11,pp. 4081-4086. https://doi.org/10.1021/ nl802408y

[13] K.L. Kavanagh, I. Saveliev, M. Blumin, G. Swadener and H.E. Ruda, Faster Radial Strain Relaxation in InAs-GaAs Core-Shell Heterowires, J. Appl. Phys., 2012, vol. 111, no. 4, art. 044301. https://doi.org/10.1063/1.3684964

[14] G. Perillat-Merceroz, R. Thierry, P.-H. Jouneau, P. Ferret and G. Feuillet, Strain Relaxation by Dislocation Glide in ZnO/ZnMgO Core-Shell Nanowires, Appl. Phys. Lett., 2012, vol. 100, no. 17, art. 173102. https://doi.org/10.1063/1.4704927

[15] S. Lee, A. Vaid, J. Im, B. Kim, A. Prakash, J. Guénolé, D. Kiener, E. Bitzek and S.H. Oh, Insitu Observation of the Initiation of Plasticity by Nucleation of Prismatic Dislocation Loops, Nat. Commun., 2020, vol. 11, no. 1, art. 2367. https://doi.org/10.1038/s41467-020-15775-y

[16] E. Ertekin, P.A. Greaney, D.C. Chrzan and T.D. Sands, Equilibrium Limits of Coherency in Strained Nanowire Heterostructures, J. Appl. Phys., 2005, vol. 97, no. 11, art. 114325. https://doi.org/10.1063/1.1903106

[17] F. Glas, Critical Dimensions for the Plastic Relaxation of Strained Axial Heterostructures in Free-Standing Nanowires, Phys. Rev. B., 2006, vol. 74, no. 12, art. 121302. https://doi.org/ 10.1103/PhysRevB.74.121302

[18] A. Arumbakkam, E. Davidson and A. Strachan, Heteroepitaxial Integration of Metallic Nanowires: Transition from Coherent to Defective Interfaces via Molecular Dynamics, Nanotechnology, 2007, vol. 18, no. 34, art. 345705. https://doi.org/10.1088/0957-4484/18/34/345705 
[19] M. de la Mata, C. Magén, P. Caroff and J. Arbiol, Atomic Scale Strain Relaxation in Axial Semiconductor III-V Nanowire Heterostructures, Nano Lett., 2014, vol. 14, no. 11,pp. 6614-6620. https://doi.org/10.1021/ nl503273j

[20] H. Ye and Z. Yu, Plastic Relaxation of Mixed Dislocation in Axial Nanowire Heterostructures Using Peach-Koehler Approach, Phys. Status Solidi - Rapid Res. Lett., 2014, vol. 8, no. 5, pp. 445-448. https://doi.org/10.1002/pssr.201409072

[21] F. Glas, Strain in Nanowires and Nanowire Heterostructures, In: Semicond. Nanowires I Growth Theory, Semicond. Semimetals, ed. by A.F.I. Morral, S.A. Dayeh and C. Jagadish (Elsevier Inc., 2015), pp. 79-123. https://doi.org/ 10.1016/bs.semsem.2015.09.004

[22] X. Kong, S. Albert, A. Bengoechea-Encabo, M.A. Sanchez-Garcia, E. Calleja and A. Trampert, Lattice Pulling Effect and Strain Relaxation in Axial (In,Ga)N/GaN Nanowire Heterostructures Grown on GaN-Buffered Si(111) Substrate, Phys. Status Solidi A, 2015, vol. 212, no. 4, pp. 736-739. https://doi.org/10.1002/pssa.201400198

[23] M.Yu. Gutkin, I.A. Ovid'ko and A.G. Sheinerman, Misfit Dislocations in Wire Composite Solids, J. Phys. Condens. Matter., 2000, vol. 12, no. 25, pp. 5391-5401. https://doi.org/10.1088/0953-8984/ $12 / 25 / 304$

[24] A.G. Sheinerman and M.Yu. Gutkin, Misfit Disclinations and Dislocation Walls in a TwoPhase Cylindrical Composite, Phys. Status Solidi A, 2001, vol. 184, no. 2, pp. 485-505. https:/ /doi.org/10.1002/1521-396X(200104)184:2<485: :AID-PSSA485>3.0.CO;2-4

[25] H.-M. Lin, Y.-L. Chen, J. Yang, Y.-C. Liu, K.-M. Yin, J.-J. Kai, F.-R. Chen, L.-C. Chen, Y.-F. Chen and C.-C. Chen, Synthesis and Characterization of Core-ShellGaP@GaN and GaN@GaP Nanowires, Nano Lett., 2003, vol. 3, no. 4, pp. 537-541. https://doi.org/10.1021/n10340125

[26] I.A. Ovid'ko and A.G. Sheinerman, Misfit Dislocation Loops in Composite Nanowires, Philos. Mag., 2004, vol. 84, pp. 2103-2118. https://doi.org/10.1080/14786430410001678163

[27] S. Raychaudhuri and E.T. Yu, Critical Dimensions in Coherently Strained Coaxial Nanowire Heterostructures, J. Appl. Phys., 2006, vol. 99, no. 11, art. 114308. https://doi.org/10.1063/1.2202697

[28] I.A. Ovid'ko and A.G. Sheinerman, Misfit Dislocations in Nanocomposites with Quantum Dots, Nanowires and their Ensembles, Adv. Phys., 2006, vol. 55, nos. 7-8, pp. 627-689. https://doi.org/10.1080/00018730600976684
[29] K.E. Aifantis, A.L. Kolesnikova and A.E. Romanov, Nucleation of Misfit Dislocations and Plastic Deformation in Core/Shell Nanowires, Philos. Mag., 2007, vol. 87, no. 30, pp. 4731-4757. https://doi.org/10.1080/14786430701589350

[30] I.A. Goldthorpe, A.F. Marshall and P.C. McIntyre, Inhibiting Strain-Induced Surface Roughening: Dislocation-Free Ge/Si and Ge/SiGe Core-Shell Nanowires, Nano Lett., 2009, vol. 9, no. 11, pp. 3715-3719. https://doi.org/10.1021/n19018148

[31] J. Colin, Prismatic Dislocation Loops in Strained Core-Shell Nanowire Heterostructures, Phys. Rev. B, 2010, vol. 82, no. 5, art. 054118. https:// doi.org/10.1103/PhysRevB.82.054118

[32] X. Wang, E. Pan and P.W. Chung, Misfit Dislocation Dipoles in Wire Composite Solids, Int. J. Plast., 2010, vol. 26, no. 9, pp. 1415-1420. https://doi.org/10.1016/j.ijplas.2010.03.004

[33] H.J. Chu, J. Wang, C.Z. Zhou and I.J. Beyerlein, Self-Energy of Elliptical Dislocation Loops in Anisotropic Crystals and its Application for Defect-Free Core/Shell Nanowires, Acta Mater., 2011, vol. 59, no. 18, pp. 7114-7124. https://doi.org/10.1016/j.actamat.2011.07.066

[34] M.Yu. Gutkin, K.V. Kuzmin and A.G. Sheinerman, Misfit Stresses and Relaxation Mechanisms in a Nanowire Containing a Coaxial Cylindrical Inclusion of Finite Height, Phys. Status Solidi B, 2011, vol. 248, no. 7, pp. 1651-1657. https://doi.org/10.1002/pssb.201046452

[35] K.L. Kavanagh, J. Salfi, I. Savelyev, M. Blumin and H.E. Ruda, Transport and Strain Relaxation in Wurtzite InAs-GaAs Core-Shell Heterowires, Appl. Phys. Lett., 2011, vol. 98, no. 15, art. 152103. https://doi.org/10.1063/1.3579251

[36] R. Popovitz-Biro, A. Kretinin, P. Von Huth and H. Shtrikman, InAs/GaAs Core-Shell Nanowires, Cryst. Growth Des., 2011, vol. 11, no. 9, pp. 3858-3865. https://doi.org/10.1021/cg200393y

[37] C.M. Haapamaki, J. Baugh and R.R. LaPierre, Critical Shell Thickness for InAs-Al $\operatorname{In}_{1-x} A s(P)$ Core-Shell Nanowires, J. Appl. Phys., 2012, vol. 112, no. 12, art. 124305. https://doi.org/10.1063/ 1.4769735

[38] S.G. Ghalamestani, M. Heurlin, L.-E. Wernersson, S. Lehmann and K.A. Dick, Growth of InAs/InP Core-Shell Nanowires with Various Pure Crystal Structures, Nanotechnology, 2012, vol. 23, no. 28, art. 285601. https://doi.org/10.1088/ 0957-4484/23/28/285601

[39] A. Nie, J. Liu, Q. Li, Y. Cheng, C. Dong, W. Zhou, P. Wang, Q. Wang, Y. Yang, Y. Zhu, Y. Zeng and H. Wang, Epitaxial $\mathrm{TiO}_{2} / \mathrm{SnO}_{2}$ Core-Shell Heterostructure by Atomic Layer Deposition, 
J. Mater. Chem., 2012, vol. 22, no. 21, pp.

10665-10671. https://doi.org/10.1039/c2jm30690e

[40] A. Biermanns, T. Rieger, G. Bussone, U. Pietsch,

D. Grützmacher and M.I. Lepsa, Axial Strain in

GaAs/InAs Core-Shell Nanowires, Appl. Phys.

Lett., 2013, vol. 102, no. 4, art. 043109.

http://dx.doi.org/10.1063/1.4790185

[41] B.T. Sneed, C.N. Brodsky, C.H. Kuo, L.K.

Lamontagne, Y. Jiang, Y. Wang, F. Tao, W. Huang and C.K. Tsung, Nanoscale-Phase-Separated Pd-Rh Boxes Synthesized via Metal Migration: An Archetype for Studying Lattice Strain and Composition Effects in Electrocatalysis, J. Am. Chem. Soc., 2013, vol. 135, no. 39, pp. 14691-14700. https://doi.org/10.1021/ja405387q

[42] S.A. Dayeh, W. Tang, F. Boioli, K.L. Kavanagh, H. Zheng, J. Wang, N.H. Mack, G. Swadener, J.Y. Huang, L. Miglio, K.-N. Tu and S.T. Picraux, Direct Measurement of Coherency Limits for Strain Relaxation in Heteroepitaxial Core/Shell Nanowires, Nano Lett., 2013, vol. 13, no. 5, pp. 1869-1876. https://doi.org/10.1021/nl3022434

[43] O. Salehzadeh, K.L. Kavanagh and S.P. Watkins, Growth and Strain Relaxation of GaAs and GaP Nanowires with GaSb Shells, J. Appl. Phys., 2013, vol. 113, no. 13, art. 134309. https://doi.org/ 10.1063/1.4799065

[44] O. Salehzadeh, K.L. Kavanagh and S.P. Watkins, Geometric Limits of Coherent III-V Core/Shell Nanowires, J. Appl. Phys., 2013, vol. 114, no. 5, art. 054301. https://doi.org/10.1063/1.4816460

[45] Y.X. Zhao, Q.H. Fang and Y.W. Liu, Edge Misfit Dislocations in Core-Shell Nanowire with Surface/Interface Effects and Different Elastic Constants, Int. J. Mech. Sci., 2013, vol. 74, pp. 173-184. https://doi.org/10.1016/ j.ijmecsci.2013.05.013

[46] M.Yu. Gutkin and A.M. Smirnov, Initial Stages of Misfit Stress Relaxation by Rectangular Prismatic Dislocation Loops in Composite Nanostructures, J. Phys. Conf. Ser., 2014, vol. 541, no. 1, art. 012007. https://doi.org/10.1088/ 1742-6596/541/1/012007

[47] M.Yu. Gutkin, S.A. Krasnitckii, A.M. Smirnov, A.L. Kolesnikova and A.E. Romanov, Dislocation Loops in Solid and Hollow Semiconductor and Metal Nanoheterostructures, Phys. Solid State, 2015, vol. 57, no. 6, pp. 1177-1182. https://doi.org/ 10.1134/S1063783415060153

[48] M.Yu. Gutkin and A.M. Smirnov, Initial Stages of Misfit Stress Relaxation in Composite Nanostructures through Generation of Rectangular Prismatic Dislocation Loops, Acta
Mater., 2015, vol. 88, pp. 91-101. https://doi.org/

10.1016/j.actamat.2015.01.020

[49] C. Enzevaee, M.Y. Gutkin and H.M. Shodja, Surface/Interface Effects on the Formation of Misfit Dislocation in a Core-Shell Nanowire, Philos. Mag., 2014, vol. 94, no. 5, pp. 492-519. https://doi.org/10.1080/14786435.2013.856527

[50] J. Colin, Circular Dislocation Loop in a ThreeLayer Nanowire, Int. J. Solids Struct., 2015, vol. 63, pp. 114-120. https://doi.org/10.1016/ j.ijsolstr.2015.02.045

[51] B.M. Nguyen, B. Swartzentruber, Y.G. Ro and S.A. Dayeh, Facet-Selective Nucleation and Conformal Epitaxy of Ge Shells on Si Nanowires, Nano Lett., 2015, vol. 15, no. 11, pp. 7258-7264. https://doi.org/10.1021/ acs.nanolett.5b02313

[52] M.B. Katz, M.E. Twigg and S.M. Prokes, Formation and Stability of Crystalline and Amorphous $\mathrm{Al}_{2} \mathrm{O}_{3}$ Layers Deposited on $\mathrm{Ga}_{2} \mathrm{O}_{3}$ Nanowires by Atomic Layer Epitaxy, J. Appl. Phys., 2016, vol. 120, no. 12, art. 124311. https://doi.org/10.1063/1.4963282

[53] S.A. Krasnitckii, A.M. Smirnov and M.Yu. Gutkin, Misfit Stresses in a Core-Shell Nanowire with Core in the Form of Long Parallelepiped, J. Phys. Conf. Ser., 2016, vol. 690, art. 012022. https://doi.org/10.1088/1742-6596/690/1/012022

[54] M.Yu. Gutkin and A.M. Smirnov, Initial Stages of Misfit Stress Relaxation through the Formation of Prismatic Dislocation Loops in $\mathrm{GaN}-\mathrm{Ga}_{2} \mathrm{O}_{3}$ Composite Nanostructures, Phys. Solid State, 2016, vol. 58, no. 8, pp. 1611-1621. https://doi.org/10.1134/S1063783416080138

[55] S.A. Krasnitckii, D.R. Kolomoetc, A.M. Smirnov and M.Yu. Gutkin, Misfit Stresses in a Composite Core-Shell Nanowire with an Eccentric Parallelepipedal Core Subjected to OneDimensional Cross Dilatation Eigenstrain, J. Phys. Conf. Ser., 2017, vol. 816, art. 012043. https://doi.org/10.1088/1742-6596/816/1/012043

[56] R.B. Lewis, L. Nicolai, H. Küpers, M. Ramsteiner, A. Trampert and L. Geelhaar, Anomalous Strain Relaxation in Core-Shell Nanowire Heterostructures via Simultaneous Coherent and Incoherent Growth, Nano Lett., 2017, vol. 17, no. 1, pp. 136-142. https://doi.org/10.1021/ acs.nanolett.6b03681

[57] Y.C. Lin, D. Kim, Z. Li, B.M. Nguyen, N. Li, S. Zhang and J. Yoo, Strain-Induced Structural Defects and their Effects on the Electrochemical Performances of Silicon Core/Germanium Shell Nanowire Heterostructures, Nanoscale, 2017, 
vol. 9, no. 3, pp. 1213-1220. https://doi.org/ $10.1039 /$ c6nr07681e

[58] S.A. Krasnitckii, D.R. Kolomoetc, A.M. Smirnov and M.Yu. Gutkin, Misfit Stress Relaxation in Composite Core-Shell Nanowires with parallelepiped Cores Using Rectangular Prismatic Dislocation Loops, J. Phys. Conf. Ser., 2018, vol. 993, no. 1, art. 012021. https://doi.org/ 10.1088/1742-6596/993/1/012021

[59] A.M. Smirnov, E.C. Young, V.E. Bougrov, J.S. Speck and A.E. Romanov, Stress Relaxation in Semipolar and Nonpolar III-Nitride Heterostructures by Formation of Misfit Dislocations of Various Origin, J. Appl. Phys., 2019, vol. 126, art. 245104. https://doi.org/10.1063/ 1.5126195

[60] S.A. Krasnitckii, A.M. Smirnov and M.Yu. Gutkin, Axial Misfit Stress Relaxation in Core-Shell Nanowires with Polyhedral Cores through the Nucleation of Misfit Prismatic Dislocation Loops, J. Mater. Sci., 2020, vol. 55, no. 22, pp. 9198-9210. https://doi.org/10.1007/s10853-02004401-3

[61] A.M. Smirnov, S.A. Krasnitckii and M.Yu. Gutkin, Generation of Misfit Dislocations in a CoreShell Nanowire near the Edge of Prismatic Core, Acta Mater., 2020, vol. 186, pp. 494-510. https://doi.org/10.1016/j.actamat.2020.01.018

[62] S.A. Krasnitckii, A.M. Smirnov and M.Yu. Gutkin, Pair Interaction of Coaxial Circular Prismatic Dislocation Loops in Elastic Solids with Spherical Surfaces, Mater. Phys. Mech., 2020, vol. 44, no. 1,pp. 116-124. https://doi.org/ 10.18720/MPM.4412020_14

[63] A.P. Chernakov, A.L. Kolesnikova, M.Yu. Gutkin and A.E. Romanov, Periodic Array of Misfit Dislocation Loops and Stress Relaxation in Core-Shell Nanowires, Int. J. Eng. Sci., 2020, vol. 156, art. 103367. https://doi.org/10.1016/ j.ijengsci.2020.103367

[64] F.C. Frank and J.H. Van der Merwe, OneDimensional Dislocations. I. Static Theory, Proc. Roy. Soc. London A, 1949, vol. 198, pp. 205-225. https://doi.org/10.1098/rspa.1949.0095

[65] J.W. Matthews, Defects Associated with the Accommodation of Misfit between Crystals, J. Vac. Sci. Technol., 1975, vol. 12, no. 1, pp. 126-133. http://dx.doi.org/10.1116/1.568741

[66] V.I. Vladimirov, M.Yu. Gutkin and A.E. Romanov, Influence of Free Surface on Equiponderant Stress State in Heteroepitaxial Systems, Poverkhnost'. Fizika, khimiya, mekhanika (USSR), 1988, no. 6, pp. 46-51, In Russian.
[67] M.Yu. Gutkin and A.E. Romanov, States of the Interfacial Defect Structures in Thin-Film Heterosystems, Sov. Phys. - Solid State (USA), 1990, vol. 32, no. 5, pp. 751-753.

[68] J.R. Willis, S.C. Jain and R. Bullough, The Energy of an Array of Dislocations: Implications for Strain Relaxation in Semiconductor Heterostructures, Philos. Mag. A, 1990, vol. 62, no. 1, pp. 115-129. https://doi.org/10.1080/ 01418619008244339

[69] A. Rocket and C.J. Kiely, Energetics of Misfit- and Threading-Dislocation Arrays in Heteroepitaxial Films, Phys. Rev. B, 1991, vol. 44, no. 3, pp. 1154-1162. https://doi.org/10.1103/ PhysRevB.44.1154

[70] A. Atkinson and S.C. Jain, The Energy of Systems of Misfit Dislocations in Epitaxial Strained Layers, Thin Solid Films, 1992, vol. 222, no. 1-2, pp. 161-165. https://doi.org/10.1016/00406090(92)90060-O

[71] A. Atkinson and S.C. Jain, The Energy of Finite Systems of Misfit Dislocations in Epitaxial Strained Layers, J. Appl. Phys., 1992, vol. 72, no. 6, pp. 2242-2248. https://doi.org/10.1063/1.351617

[72] T.J. Gosling, S.C. Jain, J.R. Willis, A. Atkinson and R. Bullough, Stable Configurations in Strained Epitaxial Layers, Philos. Mag. A, 1992, vol. 66, no. 1, pp. 119-132. https://doi.org/10.1080/ 01418619208201517

[73] M.Yu. Gutkin and A.E. Romanov, Misfit Dislocations in a Thin Two-Phase Heteroepitaxial Plate, Phys. Stat. Sol. A, 1992, vol. 129, no. 2, pp. 117-126. https://doi.org/ 10.1002/pssa.2211290109

[74] S.C. Jain, T.J. Gosling, J.R. Willis, R. Bullough and P. Balk, Theoretical Comparison of the Stability Characteristics of Capped and Uncapped Ge ${ }_{x} \mathrm{Si}_{1-x}$ Strained Epilayers, Solid-State Electronics, 1992, vol. 35, no. 8, pp. 1073-1079. https://doi.org/10.1016/0038-1101(92)90007-Y

[75] S.C. Jain, T.J. Gosling, J.R. Willis, D.H.J. Totterdell and R. Bullough, A New Study of Critical Layer Thickness, Stability and Strain Relaxation in Pseudomorphic Ge Si $_{1-x}$ Strained Epilayers, Philos. Mag. A, 1992, vol. 65, no. 5, pp. 11511167. https://doi.org/10.1080/01418619208201502

[76] T.J. Gosling, R. Bullough, S.C. Jain and J.R. Willis, Misfit Dislocation Distributions in Capped (Buried) Strained Semiconductor Layers, J.Appl. Phys., 1993, vol. 73, no. 12, pp. 8267-8268. https://doi.org/10.1063/1.353445

[77] M.Yu. Gutkin, A.L. Kolesnikova and A.E. Romanov, Misfit Dislocations and Other Defects 
in Thin Films, Mater. Sci. Eng. A, 1993, vol. 164, nos. 1-2, pp. 433-437. https://doi.org/10.1016/ 0921-5093(93)90707-L

[78] U. Jain, S.C. Jain, J. Nijs, J.R. Willis, R. Bullough, R.P. Mertens and R. Van Oversraeten, Calculation of Critical-Layer-Thickness and Strain Relaxation in $\mathrm{Ge}_{x} S i_{1-x}$ Strained Layers with Interacting 60 and $90^{\circ}$ Dislocations, Solid State Electronics, 1993, vol. 36, no. 3, pp. 331-337. https://doi.org/10.1016/0038-1101(93)90084-4

[79] T.J. Gosling and J.R. Willis, The Energy of Arrays of Dislocations in an Anisotropic Half-Space, Philos. Mag. A, 1994, vol. 69, no. 1, pp. 65-90. https://doi.org/10.1080/01418619408242211

[80] F. Bailly, M. Barbé and G. Cohen-Solal, Setting up of Misfit Dislocations in Heteroepitaxial Growth and Critical Thicknesses, J. Cryst. Growth, 1995, vol. 153, nos. 3-4, pp. 115-122. https://doi.org/ 10.1016/0022-0248(95)00211-1

[81] M.Yu. Gutkin, K.N. Mikaelyan and I.A. Ovid'ko, Equilibrium Configurations of Partial Misfit Dislocations in Thin-Film Heterosystems, Phys. Solid State, 1998, vol. 40, no. 11, pp. 1864-1869. https://doi.org/10.1134/1.1130674

[82] Yu.A. Tkhorik and L.S. Khazan, Plastic Deformation and Misfit Dislocations in Heteroepitaxial Systems (Naukova Dumka, Kiev, 1983), In Russian.

[83] L.B. Freund and S. Suresh, Thin Film Materials. Stress. Defect Formation and Surface Evolution (Cambridge University Press, Cambridge, UK, 2003).

[84] M.Yu. Gutkin and I.A. Ovid'ko, Physical Mechanics of Deformed Nanostructures. Vol. II. Nanolayered Structures (Yanus, SaintPetersburg, 2005), In Russian.

[85] M.Yu. Gutkin, Strength and Plasticity of Nanocomposites: Textbook (Izdatel'stvo Politekhnicheskogo universiteta, 2011), In Russian.

[86] H.M. Shodja, C. Enzevaee and M.Yu. Gutkin, Interface Effect on the Formation of a Dipole of Screw Misfit Dislocations in an Embedded Nanowire with Uniform Shear Eigenstrain Field, European Journal of Mechanics - A / Solids, 2015, vol. 51, no. 1, pp. 154-159. https://doi.org/10.1016/j.euromechsol.2014.12.006

[87] J.W. Gibbs, The Scientific Papers of J. Willard Gibbs, Vol. 1 (Longmans Green, London, 1906).

[88] R. Shuttleworth, The Surface Tension of Solids, Proc. Phys. Soc. A, 1950, vol. 63, pp. 445-458. https://doi.org/10.1088/0370-1298/63/5/302

[89] M.E. Gurtin and A.I. Murdoch, A Continuum Theory of Elastic Material Surfaces, Arch.
Rational Mech. Anal., 1975, vol. 57, pp. 291-323. https://doi.org/10.1007/BF00261375

[90] M.E. Gurtin and A.I. Murdoch, Surface Stress in Solids, Int. J. Solids Struct., 1978, vol. 14, pp. 431-440. https://doi.org/10.1016/00207683(78)90008-2

[91] R.C. Cammarata, Surface and Interface Stress Effects in Thin Film, Prog. Surf. Sci., 1994, vol. 46, pp. 1-38. https://doi.org/10.1016/00796816(94)90005-1

[92] M.E. Gurtin, J. Weissmuller and F. Larché, A General Theory of Curved Deformable Interfaces in Solids at Equilibrium, Phil. Mag. A, 1998, vol. 78, pp. 1093-1109. https://doi.org/ 10.1080/01418619808239977

[93] H. Altenbach, V.A. Eremeyev and N.F. Morozov, On Equations of the Linear Theory of Shells with Surface Stresses Taken into Account, Mechanics of Solids, 2010, vol. 45, pp. 331-342. https://doi.org/10.3103/S0025654410030040

[94] R.V. Goldstein, V.A. Gorodtsov and K.B. Ustinov, Effect of Residual Surface Stress and Surface Elasticity on Deformation of Nanometer Spherical Inclusions in an Elastic Matrix, Phys. Mesomech., 2010, vol. 13, pp. 318-328. https://doi.org/10.1016/j.physme.2010.11.012

[95] M.A. Grekov and T.S. Sergeeva, Interaction of Edge Dislocation Array with Bimaterial Interface Incorporating Interface Elasticity, Int. J. Engng. Sci., 2020, vol. 149, pp. 103233(1)103233(17). https://doi.org/10.1016/ j.ijengsci.2020.103233

[96] P. Sharma and S. Ganti, Size-Dependent Eshelby's Tensor for Embedded Nano-Inclusions Incorporating Surface/Interface Energies, J. Appl. Mech., 2004, vol. 71, pp. 663-671. https://doi.org/10.1115/1.1781177

[97] P. Sharma, S. Ganti and N. Bhate, Effect of Surfaces on Size-Dependent Elastic State of Nanoinhomogeneities, Appl. Phys. Lett., 2003, vol. 82, pp. 535-537. https://doi.org/10.1063/ 1.1539929

[98] L. Tian and R.K.N.D. Rajapakse, Elastic Field of an Isotropic Matrix with a Nanoscale Elliptical Inhomogeneity, Int. J. Solids Struct., 2007, vol. 44, pp. 7988-8005. https://doi.org/10.1016/ j.ijsolstr.2007.05.019

[99] S. Timoshenko and J.N. Goodier, Theory of Elasticity (McGraw-Hill Book Co., New York / Toronto / London, 1951).

[100] I.A. Ovid'ko and A.G. Sheinerman, Mechanics of Nanowires and Nanostructured Films ( ExlibrisNord, Saint-Petersburg, 2011), In Russian. 
[101] A.L. Kolesnikova and A.E. Romanov, Dislocation and Disclination Loops in the Virtual-Defect Method, Phys. Solid State, 2003, vol. 45, no. 9, pp. 1706-1718. https://doi.org/ $10.1134 / 1.1611238$

[102] A.L. Kolesnikova and A.E. Romanov, Virtual Circular Dislocation-Disclination Loop Technique in Boundary Value Problems in the Theory of Defects, J. Appl. Mech., 2004, vol. 71, no. 3, pp. 409-417. https://doi.org/10.1115/ 1.1757488

[103] M.Yu. Gutkin, M. Militzer, A.E. Romanov and V.I. Vladimirov, Equilibrium Position of Misfit Dislocations, Phys. Stat. Sol. (a), 1989, vol. 113, no. 2, pp. 337-344. https://doi.org/10.1002/ pssa.2211130211

[104] M.Yu. Gutkin and A.E. Romanov, Misfit Dislocations in a Thin Two-Phase Heteroepitaxial Plate, Phys. Stat. Sol. (a), 1992, vol. 129, no. 2, pp. 117-126. https://doi.org/ 10.1002/pssa.2211290109

[105] M.Yu. Gutkin and A.E. Romanov, On the Standoff Positions of Misfit Dislocations, Phys. Stat. Sol. (a), 1994, vol. 144, no. 1, pp. 39-57. https://doi.org/10.1002/pssa.2211440106

[106] A.I. Lurie, Spatial Problems of Theory of Elasticity (State Publishing House of Scientific and Technical Literature, Moscow, 1955), In Russian.

[107] M.Y. Gutkin, I.A. Ovid'ko and A.G. Sheinerman, Misfit Dislocations in Composites with Nanowires, J. Physics - Condensed Matter.,
2003, vol. 15, no. 21, pp. 3539-3554. https://doi.org/10.1088/0953-8984/15/21/304

[108] M.Yu. Gutkin, Misfit Stress Relaxation in Composite Nanoparticles, Intern. J. Eng. Sci., 2012, vol. 61 (Special Issue), pp. 59-74. https://doi.org/10.1016/j.ijengsci.2012.06.010

[109] M.Yu. Gutkin, A.L. Kolesnikova, S.A. Krasnitckii and A.E. Romanov, Misfit Dislocation Loops in Composite Core-Shell Nanoparticles, Phys. Solid State, 2014, vol. 56, no. 4, pp. 723-730. https://doi.org/10.1134/S1063783414040106

[110] M.Yu. Gutkin, A.L. Kolesnikova, S.A. Krasnitckii, A.E. Romanov and A.G. Shalkovskii, Misfit Dislocation Loops in Hollow Core-Shell Nanoparticles, Scripta Materialia, 2014, vol. 83, no. 1, pp. 1-4. https://doi.org/10.1016/ j.scriptamat.2014.03.005

[111] M.Yu. Krauchanka, S.A. Krasnitckii, M.Yu. Gutkin, A.L. Kolesnikova and A.E. Romanov, Circular Loops of Misfit Dislocations in Decahedral Core-Shell Nanoparticles, Scripta Materialia, 2019, vol. 167, pp. 81-85. https://doi.org/10.1016/j.scriptamat.2019.03.031

[112] M.Yu. Gutkin and A.M. Smirnov, Generation of Rectangular Prismatic Dislocation Loops in Shells and Cores of Composite Nanoparticles, Phys. Solid State, 2014, vol. 56, no. 4, pp. 731738. https://doi.org/10.1134/S1063783414040118

[113] I.A. Ovid'ko and A.G. Sheinerman, Perfect, Partial, and Split Dislocations in Quantum Dots, Phys. Rev. B, 2002, vol. 66, art. 245309. https://doi.org/10.1103/PhysRevB.66.245309 